\title{
Les lœss en France et dans le Nord-Ouest européen
}

\section{P. ANTOINE}

CNRS ESA 8018,

Unité Stratigraphie et Paléoenvironnements quaternaires

UFR de Géographie, Université des Sciences et Technologies de Lille avenue Paul-Langevin 59655 Villeneuve-d'Ascq Cedex Pierre.Antoine@univ-lille1.fr
Les løess sont des limons d'origine éolienne

caractéristiques des environnements périglaciaires et des maxima glaciaires du Quaternaire (depuis 2,4 Ma). Ils se localisent essentiellement entre 30 et $60^{\circ}$ de latitude Nord, en marge des grands inlandsis et des zones désertiques (Chine). Les alternances loess-paléosols constituent des séquences lœssiques dont l'épaisseur, de l'ordre de 5 à $40 \mathrm{~m}$ en Europe occidentale et centrale, peut localement dépasser $200 \mathrm{~m}$ en Chine où ils correspondent à environ 2,4 Ma. Actuellement, l'étude multidisciplinaire de ces séquences est un des moyens privilégiés de reconstitution de l'enregistrement paléoclimatique en domaine continental. Au sein de ces séquences, les lœess typiques sont constitués par des silts quartzeux (fraction dominante: $10-50 \mu \mathrm{m}$ ), calcaires, extrèmement bien classés, pauvres en sable, non stratifiés, et qui possèdent une structure isotrope fortement poreuse et métastable. Du point de vue géotechnique, ce sont des matériaux effondrables, extrêmement sensibles aux variations d'humidité, à la compaction et aux vibrations. Dans le Nord-Ouest de l'Europe, oủ ils constituent une zone plus ou moins continue de la Bretagne à la vallée du Rhin, l'analyse détaillée de la séquence du Pléistocène supérieur $( \pm 128-15 \mathrm{ka}$ BP) permet de mettre en évidence une succession pédosédimentaire complexe au sein de laquelle les lœss carbonatés sont caractéristiques d'une courte période centrée autour du dernier maximum glaciaire (30-15 ka BP). Enfin, le développement des grands travaux d'aménagement dans le domaine lœssique de la France septentrionale (TGV Nord, autoroutes, gazoducs...), permet de souligner l'importance de la prise en compte de la variabilité des faciès loessiques pour une meilleure approche des problèmes géotechniques liés à ces matériaux.

Mots-clés : lœess, Europe du Nord-Ouest, paléosols, environnements périglaciaires, stratigraphie, sédimentologie.
The loess are aeolian silts which are typical of periglacial environments and of the full Glacial periods of the Quaternary. They are mainly located between 30 and $60^{\circ} \mathrm{N}$ at the margin of the main icecaps and of desert zones (China). The superimposition of loess and palaeosols are named loess sequences or loess series. Their thickness varies from 5 to $40 \mathrm{~m}$ in Western and Central Europe and locally up to $200 \mathrm{~m}$ in China where they represent about $2,4 \mathrm{My}$. Presently their multidisciplinary investigation is one of the best way to highlight the impact of global climate changes on the continental zones.

Within these sequences, typical calcareous loess are represented by well sorted homogeneous and calcareous quartz silts (main grain-size class : $10-50 \mu \mathrm{m}$ ), showing a strongly porous, isotrope and meta-stable fabric. From a geotechnical point of view they are classified as collapsible soils and are very sensitive to changes in water content and to compaction and vibrations. In 
north-western Europe, where they represent a more or less continuous zone between Brittany and the Rhine Valley, the detailed investigation of the Upper Pleistocene sequence $( \pm 128$ 15 ky BP) provide evidence a very complex pedosedimentary record in which typical calcareous loess are typical of a short episode centred on the Last Glacial Maximum $( \pm 30-15 \mathrm{ky}$ BP). Finally, the very important development of construction works in the loess area of Northern France (highway infrastructures. high speed train, gaz pipeline...), bring to underline the importance of taking in account the variability of loess facies for a better approach of the geotechnical problems linked to these materials.

Key words : loess, North-Western Europe, palaeosols, periglacial environments, stratigraphy, sedimentology.

\section{Introduction : définition et généralités}

Les lcess sont généralement définis comme des sédiments limoneux éoliens non stratifiés, mis en place lors des périodes les plus froides du Quaternaire (phases pléniglaciaires, caractérisées par l'extension maximale des glaciers). Ils se localisent en marge des grands inlandsis comme en Amérique du Nord ou en Europe (lœess périglaciaires), ou des déserts comme en Chine (lcess péridésertiques), et représentent la formation corrélative des périodes glaciaires la plus largement représentée sur les continents (Pécsi, 1990) (Fig. 1), Ils sont presque exclusivement présents dans I'hémisphère Nord, entre 30 et $60^{\circ} \mathrm{N}$, et plus particulièrement autour du $50^{\mathrm{c}}$ parallèle, où leur répartition est conditionnée par l'extension maximale des fronts glaciaires (Fig. 1). En effet, la sédimentation lcessique est fortement conditionnée par la présence de zones source soumises à la déflation éolienne, comme les plaines de l'outwash glaciaire où se concentrent les matériaux drainés par les eaux de fonte en marge des glaciers (Fig. 2), les estuaires et les plaines alluviales continentales du domaine périglaciaire (Pye, 1984, 1987 ; Lautridou, 1985). Enfin, il faut signaler que dans les environnements actuels, des limons éoliens de faciès loessique se mettent en place localement en marge des vallées en Alaska (Péwé, 1962, 1968).

Pour la Commission du loess de l'International Union for Quaternary Research (INQUA), le loess se définit comme " un sédiment limoneux mis en place dans des conditions d'environnement périglaciaires où interviennent des processus d'érosion-sédimentation dominés par l'agent éolien, sans qu'il soit exciusif, et des processus biologiques spécifiques « lœessification ». (Jamagne et al., 1981). En croisant les caractéristiques proposées par différents auteurs (Richthofen, 1882 ; Smalley, 1971, 1975 ; Liu et al., 1985; Lautridou, 1985 ; Pécsi, 1990), il est possible de proposer une définition générale des loess typiques sur la base des critères génétiques et sédimentologiques suivants :

- origine éolienne :

- mise en place en contexte périglaciaire (froid aride à semi-aride " steppe froide $»$ ) ;

- indépendance vis-à-vis du substrat forigine allochtone, homogénéité sur de grandes distances) :

- composition granulométrique dominée par la fraction 10-50 $\mu \mathrm{m}$, courbe cumulative sigmoïde très redressée et dissymétrique, médiane de 25 à $30 \mu \mathrm{m}$, très faible pourcentage de sable ;

- composition minéralogique nettement dominée par les grains de quartz (moyenne 60 à $70 \%$ ) ;

- présence de carbonates primaires dans toutes les fractions granulométriques (maximum 20 à $25 \%$ ) : - dépôt non stratifié, homogène, à porosité élevée (40 à $55 \%$ de vides), à structure originelle isotrope et métastable.

Par ailleurs l'homogénéité des faciès sur de grandes distances n'exclut pas pour autant une diminution progressive de la médiane au fur et à mesure que l'on s'éloigne de la zone source (Sommé, 1969).

Du point de vue paléobiologique, les lœss se définissent par un contenu malacologique (mollusques) de type " faune à Pupilla ») (espèce des espaces découverts), palynologique (pollens) où dominent les herbacées, indiquant des conditions de dépôt périglaciaires dans un contexte de « steppe froide » (Lozec, 1964; Kukla, 1977 ; Rousseau, 1987, 1989, 1991).

Du point de vue géographique, les loss se répartissent sur les plateaux, les versants et dans les principaux bassins alluviaux : « lœess Plateau n en Chine où leur épaisseur peut localement atteindre $300 \mathrm{~m}$, bassin du Mississippi, plateau des løess de Sibérie, plaine russe, plaine du Danube, Rhin moyen, plateaux du Nord de la France (Figs. 1 et 5). Du point de vue morphologique, les lœess se présentent essentiellement sous la forme de couverture (continue ou discontinue), de colmatage de versant dans les vallées (en recouvrement des nappes alluviales), ou encore localement sous la forme de grandes "dunes ) allongées (lœess «Gredas ) : rive droite de la vallée du Rhin, Bavière, Bulgarie (Léger, 1990).

En dehors de la zone tempérée de l'hémisphère nord, des loess atypiques loess-like sediments sont observés dans la zone méditerranéenne et sur les bordures du Sahara (Coudé-Gaussens, 1987). Ces dépôts éoliens se différencient des løess typiques de la zone tempérée de l'hémisphère nord par des proportions d'arcile ou de sable beaucoup plus forte. Ces loess péridésertiques des bordures du Sahara sont dénommés Warm Loess par opposition aux Cold Laess mis en place dans la zone périglaciaire (Pécsi, 1990). Dans l'hémisphère sud, les lcess ne sont véritablement présents que dans le Nord de l'Argentine (Ariondo, 1990: Kröhling, 1999), et en Nouvelle-Zélande.

Par ailleurs, les loess font partie d'un ensemble plus vaste de sédiments éoliens périglaciaires auquel appartiennent aussi les sables de couverture et les dépôts sablo-limoneux de transition (Jamagne et al., 1981). La répartition géographique de ces différents faciès résulte de la zonation éolienne périglaciaire (Figs. 5 et 6), clairement mise en évidence en Europe du NordOuest et plus particulièrement dans le Nord de la France (Paepe et Sommé, 1970, Lautridou et Sommé, 1974).

Parallèlement aux loess typiques il existe un grand nombre de faciès sédimentaires lœessiques dérivés des lœess qui représentent localement une partie importante des séquences (10 à $30 \%$ ). Ils correspondent à des lœess ayant subi un remaniement post-sédimentaire comme les lœess ruisselés ou soliflués (Haesaerts, 1985 ; Vreeken et Mücher, 1981; Mücher et al., 1981). Enfin, les séquences lœssiques contiennent aussi des faciès dont la mise en place est exclusivement liée à des processus 


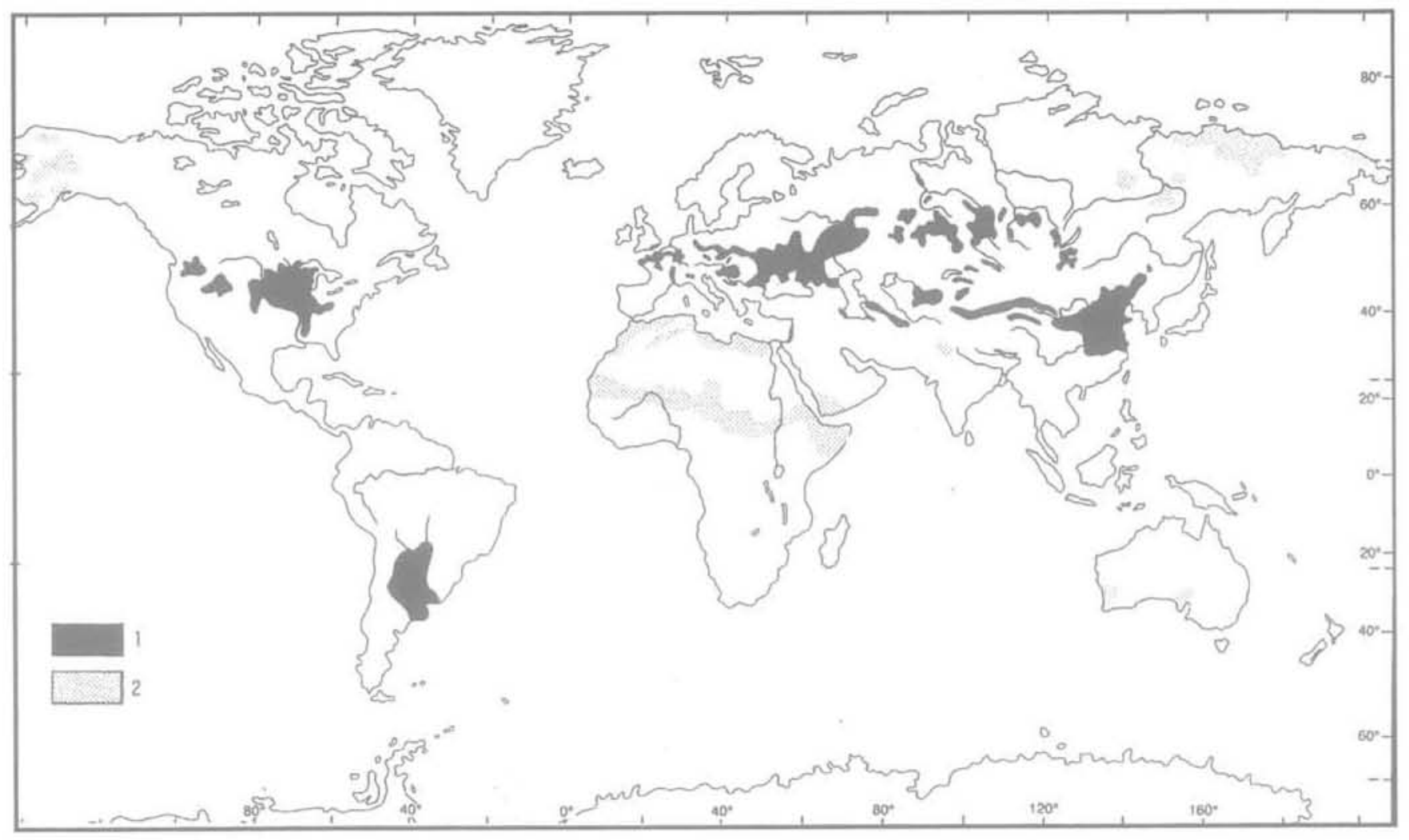

FIG.1 Répartition des lœss (1) et des sédiments éoliens apparentés (2) (d'après Pécsi, 1990). Distribution of loess (1) and « loess-liken sediments (2) (according to Pécsi, 1990).

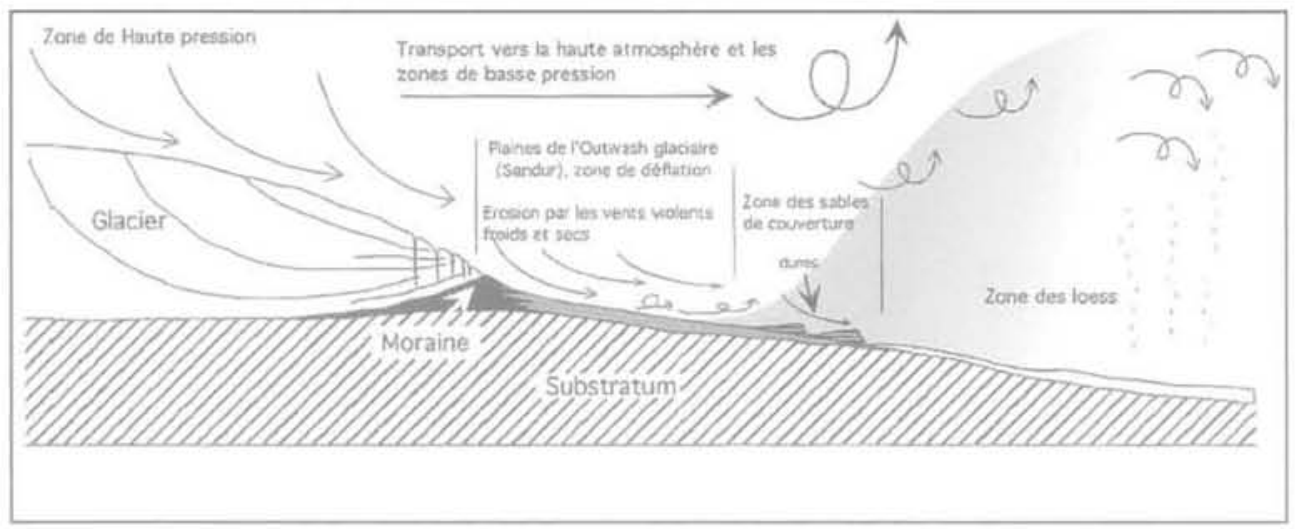

FGG.2 Modèle de mise en place des sédiments éoliens en zone périglaciaire à proximité des grands inlandsis (redessiné d'après Andersen \& Borns, 1997).

1- Les particules fines produites par l'abrasion glaciaire sont drainées et redéposées en marge des glaciers par les écoulements proglaciaires (Outwash plain).

2- Les particules sableuses, limoneuses et argileuses, soumises au gel-dégel, sont remobilisées par l'érosion éolienne (déflation), sous l'action des vents violents en provenance de la calotte, puis partiellement injectées dans la haute atmosphère oủ elles sont intégrées à la circulation dépressionnaire d'Ouest.

3- Les particules les plus grossières transportées par saltation, ou à faible altitude se déposent rapidement sous forme de dunes, puis de couverture sableuse (zone des Sables de Couverture).

4-Les particules limoneuses et argileuses transitent dans la circulation dépressionnaire puis se déposent à plus grancle distance sous l'effet de la baisse de la dynamique éolienne (chute de la vitesse du vent, obstacles, captation par la couverture herbacée ou la neige....).

Model of aeolian deposition in periglacial zones adjacent to large ice-sheets (redrawn according to Andersen $\&$ Borns, 1997).

1 - The fine particles produced by glacial erosion are washed out and redeposited at the margins of the inlandsis by proglacial flows.

2- The sandy, silty and clayey particles, subjected to freeze-thaw, are remobilized by aeolian erosion (deflation) produced by the violent winds coming from the inlandsis, then they are partially injected in the high atmosphere where they are transported by the west-wind circulation.

3 - The coarser particles transported by saltation or at low height, are quickly deposited as dunes, then as sand cover (Cover Sands zone).

4 - The silty and clayey particles are transported by the low pressure circulation then are deposited owing to a decrease of aeolian dynamic (fall in wind speed, obstacle, capture by herbaceous vegetation or snow cover). 


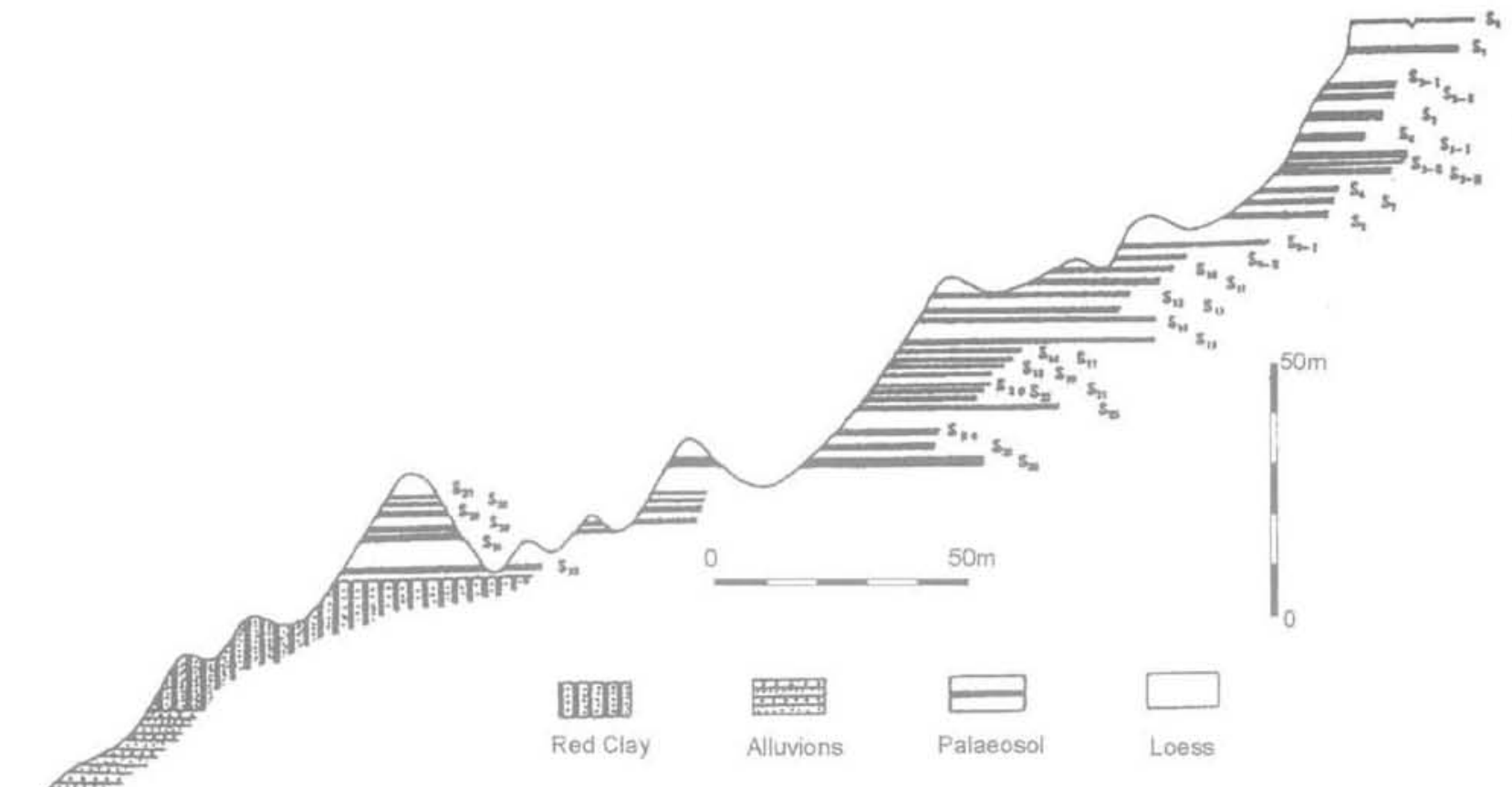

FG. 3 Coupe de la séquence løessique de Baoji. Chine (d'après Liu et al., 1991).

Cross section trough the Baoji loess and palaeosols sequence, China (according to Liu and al., 1991).

de versant : sables ruisselés, cailloutis soliflués, Lehm-brokelsands (dépôts limoneux lités à nodules de sols remaniés issus de l'érosion des sols). Ces niveaux, souvent peu épais, témoignent en général de phases d'érosion en début de dégradation climatique et se localisent préférentiellement à l'interface paléosol-lœess (Kukla, 1977).

Du point de vue stratigraphique, les différentes unités de dépôt lœessique s'organisent en séries ou séquences lcessiques qui montrent une nature fondamentalement cyclique d'origine climatique (Kukla, 1977, Liu et al., 1991). Cette cyclicité se manifeste par l'alternance, au sein de séquences lœssiques, de succession lœss-paléosols (Fig.3), correspondant globalement à des cycles climatiques de type glaciaire-interglaciaire, dont la durée est en moyenne de 100000 ans (Fig. 4).

Les nombreuses études sur la stratigraphie des lœess complétées par des données sédimentologiques, magnétostratigraphiques, et géochronologiques permettent de proposer une corrélation entre l'enregistrement climatique en domaine continental, exprimé par les séquences lœessiques, et les courbes de paléotempératures océaniques basées sur le rapport des isotopes de l'oxygène (Kukla, 1977, 1987; Liu et al., 1985; Shackleton et al., 1995; Liu et Ding, 1998). Les grandes séquences lœssiques représentent en effet l'enregistrement le plus continu des variations climatiques en domaine continental (Kukla et An, 1989; Liu, et al., 1985, 1991). Ainsi, en Chine centrale (loess Plateau), il est possible de décrire plus de 30 alternances loess-paléosol couvrant les derniers 2,4 Ma au sein de séquences qui atteignent 150 à $180 \mathrm{~m}$ d'épaisseur (Fig. 3).

Pour le dernier cycle interglaciaire-glaciaire (EemienWeichselien : 128-13 000 BP), des reconstitutions paléoclimatiques et paléoenvironnementales de plus en plus poussées sont proposées sur la base d'études paléopédologiques (Haesaerts, 1985: Van Vliet-Lanoë, 1990; Huijzer, 1993 ; Antoine et al., 1998, 1999a), palynologiques (Munaut in Antoine et al., 1994) et malacologiques (Lozec, 1964 ; Rousseau, 1989, 1991; Rousseau et Wu, 1997),
Parallèlement, dans les séquences à fort taux de sédimentation, la mesure en continu de la variation de paramètres comme la susceptibilité magnétique (Han et al., 1991; Zisheng et al., 1991 ; Kukla et al., 1990 ; Maher et Thompson, 1992; Rousseau et al., 1998; Heller et Evans, 1995), les associations malacologiques (Rousseau et Wu, 1997), le carbone organique total et le $\delta^{13} \mathrm{C}$ (Hatté et al., 1998), le ${ }^{10} \mathrm{Be}$ (Beer et al,, 1993), les rapports fer libre/fer total et $\mathrm{R}_{\mathrm{b}} / \mathrm{S}$. (Guo et al., 1996 ; Gallet et al., 1996), ou encore de la granulométrie (An et al., 1991; Porter et An, 1995 ; Xiao et al., 1995 ; Vandenberghe et al., 1997, 1998), fournit un signal paléoclimatique qu'íl est possible de comparer avec celui des courbes paléoclimatiques globales. Ces travaux débouchent depuis peu sur la mise en évidence d'oscillations climatiques millénaires corrélables avec les événements climatiques rapides (millénaires) des carottes de glace (Liu et Ding, 1998; Ding et al., 1998).

Indépendamment de cet aspect paléoclimatique, les recherches sur les løess ont montré qu'il était possible de bâtir des systèmes litho et pédostratigraphiques détaillés sur la base de l'analyse et de la corrélation des séries loessiques, notamment à l'échelle de l'Europe (Paepe et Sommé, 1970; Sommé et al., 1980; Kukla, 1977; Haesaerts, 1985; Haesaerts et al., 1981; Lautridou et Sommé, 1974 ; Lautridou et al., 1985, 1986a, b; Antoine et al., 2001). Cette démarche, essentiellement effectuée pour le dernier cycle (souvent le mieux conservé), se fonde sur la corrélation de proche en proche des séquences à l'échelle des différents domaines (Sommé et al., 1980; Antoine, 1989b). A l'intérieur d'un domaine les séquences sont subdivisées en unités lithostratigraphiques (unités de dépôt) et pédostratigraphiques (unités de sols).

La corrélation des différentes séquences à l'échelle régionale est possible en raison de la constance des faciès lœessiques des différentes unités à l'échelle d'un domaine (ex. : Nord de la France), et surtout sur la base d'horizons repères stratigraphiques que l'on peut suivre sur la longue distance. Ces horizons repères sont consti- 


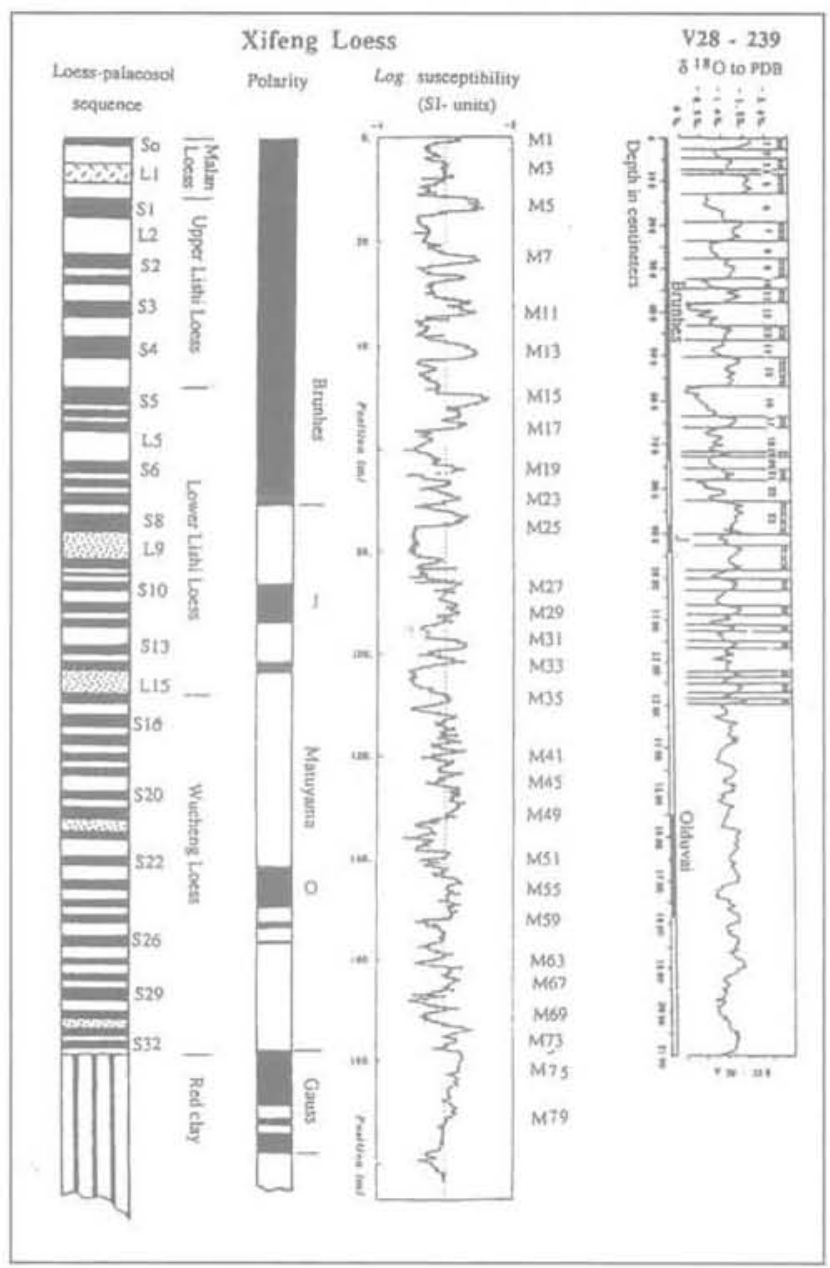

FG.4 Corrélation entre la courbe de susceptibilité magnétique de la séquence de Xifeng et la courbe de variation du $\delta^{t} \mathrm{O}$ océanique (d'après Liu et al., 1991).

Susceptibilité magnétique : mesure de la densité en minéraux ferromagnétiques des sédiments et des sols (principalement magnétite et maghémite), dont les variations sont liées à l'intensité relative de l'altération. Dans le cas général, et plus particulièrement en Chine, les loess typiques non altérés fournissent les valeurs les plus basses alors que les paléosols se caractérisent par une augmentation importante des valeurs de susceptibilité en liaison avec les processus de pédogenèse.

$\delta^{18} \mathrm{O}$ : variations du rapport entre les isotopes 16 et 18 de l'oxygène mesurés dans les carbonates des tests de foraminifères extraits des carottes océaniques profondes. Ce rapport fournit une estimation du volume global de glace stocké aux póles et des variations relatives du niveau marin.

Polarité : mesure de la polarité magnétique dans les loess qui fournit des repères précis par comparaison avec l'échelle paléomagnétique de référence,

Correlation between the magnetic susceptibility record of Chinese loess and the $\delta 180$ curve from deep-sea record (according to Liu and al., 1991).

Magnetic susceptibility: measurement of the amount of magnetic minerals in the sediments and solis (mainly magnetite and maghemite), whose the variation is linked to the relative weathering intensity. In general, and especially in China, the typical un weathered loess provide the lowest values of magnetic susceptibility, when the palaeosols are characterised by an important increase in the values, which is related to pedological processes.

$\delta^{18} \mathrm{O}$ : variations in the ratio between ${ }^{10} \mathrm{O}$ and ${ }^{110} \mathrm{O}$ measured in the carbonates of the foraminifera tests from deep sea cores. This ratio provides an assessment of the global ice volume stored on the poles and of sea level changes.

Polarity : measurement of the magnetic polarity in the loess sequence, that provides some chronological level-marks by comparison with the reference palaeomagnetic scale.

tués par des contacts stratigraphiques (surfaces d'érosion), ou des horizons pédologiques et périglaciaires (sols ou complexes de sols, niveaux de fentes de gel ou de cryoturbations), qui représentent l'expression de phénomènes climatiques globaux (Haesaerts, 1985) (Fig, 10).

Le cadre géochronologique des séquences lœssiques est fondé sur la datation par la thermoluminescence (Juvigné et Wintle, 1988; Balescu, 1988; Forman, 1991; Buraczynsky et Butrym, 1984 ; Wintle, 1987 ; Zö!ler et Wagner, 1990 ; Zöller et al., 1994 ; Frechen, 1999), les datations ${ }^{14} \mathrm{C}$ sur matériel organique (Damblon et al., 1996) ou directement sur matière organique du lœss (Hatté et al., 1998), la magnétostratigraphie (Liu et al.,
1985 ; Rutter et al., 1990 ; Heller et Evans, 1995), ou encore la racémisation des acides aminés des coquilles de mollusques (mesure du rapport entre les isomères lévogyres et dextrogyres des acides aminés, qui évolue en fonction du temps et des conditions de conservation : Oches et McCoy, 1995).

Enfin, les lœess ont donné lieu à de très nombreuses études concernant les problèmes d'effondrabilité, d'érosion et de glissement de terrain dans les régions oủ ces dépôts atteignent de fortes épaisseurs comme en Europe centrale ou en Chine (Derbyshire et al., 1991; Dijkstra, 2000) et, plus récemment, en Europe occidentale (Cui et al., 1995). 


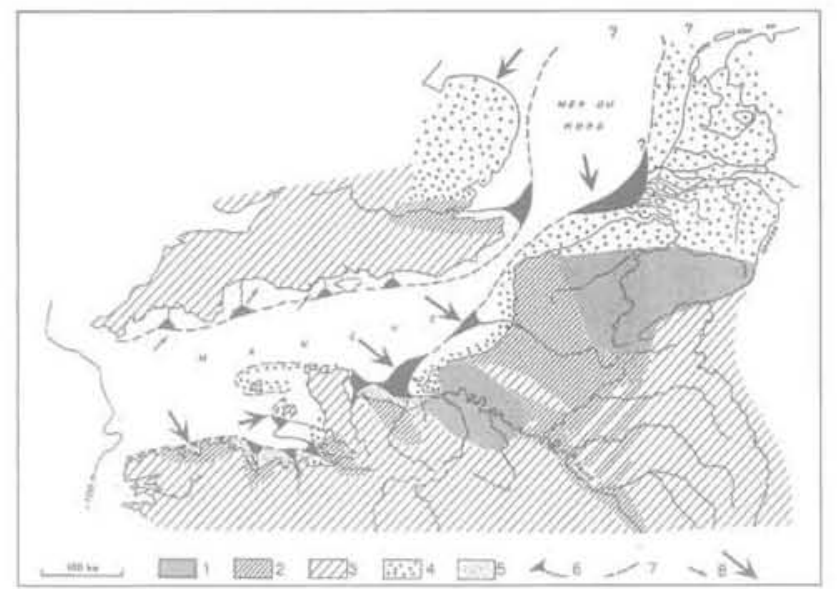

FG: 5 Carte simplifiée des couvertures lossiques et sableuses et des provinces d'alimentation pendant le Dernier glaciaire (d'après Lautridou, 1985). 1 - Loess de plus de $4 \mathrm{~m}$ d'épaisseur ; 2 Loess de 2 à $4 \mathrm{~m} ; 3$ - Loess de moins de $2 \mathrm{~m}$; 4-Sables; 5 -Loss de la plate-forme marine, submergés et érodés par la transgression holocène ; 6 Paléoestuaires : 7 - Lignes de rivage associée aux paléoestuaires : 8-Direction des vents nourriciers.

Simplified map of the lcess and sandy cover deposits and of the deflation area during the Last glacial (according to Lautridou, 1985).

1 - Loess (thickness $>4 \mathrm{~m}) ; 2$ - Loess f thickness 2 to $4 \mathrm{~m}$ ) : 3 - Loess (thickness $<2 \mathrm{~m}$ ) : 4 -Sands 5 - Loess of the marine platform, submerged and eroded by the Holocene marine extension: 6 - Palaeoestuaries: 7 - Coast lines contemporaneous of the palaeoestuaries: $8-$ Directions of the main winds.

\section{Les loess de la France septentrionale} 21

\section{Répartition}

La répartition des lcess dans la France septentrionale. telle qu'elle est actuellement cartographiée (Antoine et al., $1999 \mathrm{~b})$, correspond essentiellement à celle des loess du Pléniglaciaire supérieur weichselien ( \pm 30 -15 ka BP), dont l'extension dans le paysage masque généralement les dépôts antérieurs, d'ailleurs souvent fortement érodés.

A la suite des travaux de J. Ladrière (1890), qui fut un des premiers à présenter un système lithostratigraphique pour les limons du Nord de la France, une première tentative de cartographie des loess et limons dans l'ensemble de la France est publiée par Dubois et Firtion (1936). Plus récemment, les travaux de J.-P. Lautridou (1985) et de P. Lebret (1984) ont permis de différencier plusieurs zones dans la couverture loessique de la France septentrionale et de l'Ouest de l'Europe (Fig. 5) :

- deux zones de manteau lœssique continu : les domaines normands $(100 \times 130 \mathrm{~km})$ et nordiques $(300 \times 125 \mathrm{~km})$, où les épaisseurs moyennes sont supérieures à $4 \mathrm{~m}$. Dans sa partie septentrionale, la zone lœssique nordique passe progressivement à la zone des sables de couverture (Belgique, Pays-Bas) par l'intermédiaire de la zone de transition sablolimoneuse (Paepe et Sommé, 1974; Sommé et al., 1980) ; - des zones secondaires et marginales de faible extension
(20 à 50 km), à placages peu épais (2 à 3 m) localisées à la bordure des domaines principaux (campagne de Caen, Bocage normand, Côtes d'Armor, Kent) ;

- enfin, des placages loessiques sporadiques peu épais, de faible extension, et pollués par des altérites ou des éléments du substrat (Brie, Ardennes Champagne, Sud de l'Angleterre, Nord de la Bretagne).

Parallèlement, l'étude des variations de faciès et d'épaisseur des différents dépóts weichseliens en dans le Nord-Ouest de l'Europe a permis de mettre en évidence un passage progressif entre la zone des sables de couverture du Sud de la Belqique et la zone limoneuse du Nord de la France et de la Normandie (Paepe et Sommé 1970 Lautridou et Sommé, 1974) (Fig. 6). Plus récemment, une cartographie des lcess du Nord-Picardie a été effectuée au 1/1000 000 (Fig. 7), à l'occasion de la préparation des cartes de la France au dernier maximum glaciaire (Antoine, 1999b) ${ }^{7}$. Au sein de la zone des loess du Nord de la France, l'épaisseur totale dépasse $10 \mathrm{~m}$ (localement $25 \mathrm{~m}$ ), dans la zone orientale sur les plateaux du Hainaut, du Cambraisis et de la Picardie orientale. Dans la zone occidentale, où la couverture est moins épaisse ( 4 à $10 \mathrm{~m}$ ), les dépòts sont essentiellement contemporains du Weichselien et reposent parfois directement sur le substrat. Les dépôts du Pléistocène moyen sont plus localisés et essentiellement prèservés au sommet des nappes alluviales comme dans la Somme. Les données de sondage effectué sur le

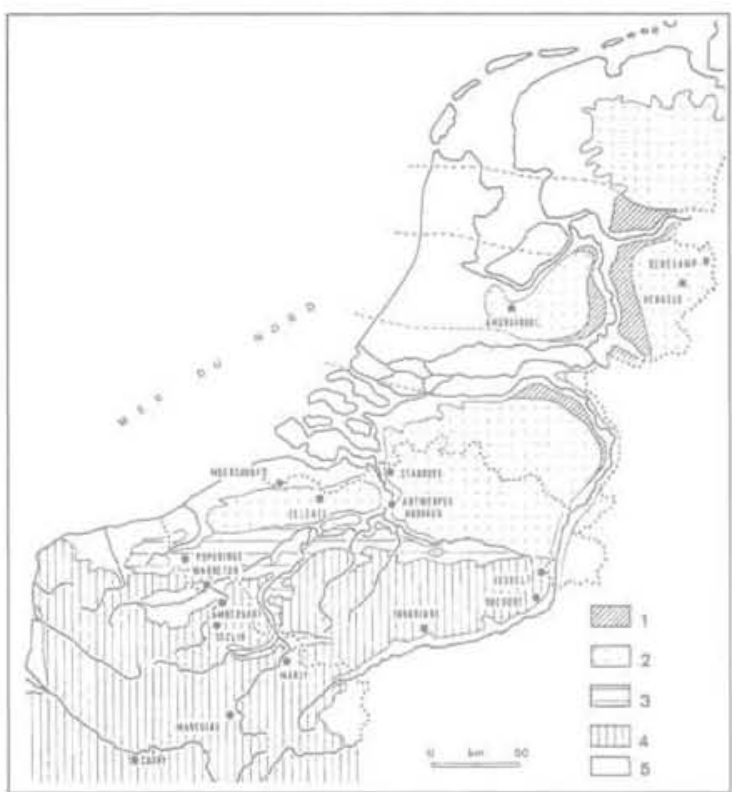

719.6 Carte du Pléistocène supérieur dans lê Nord-Ouest de l'Europe (d'après Zagwijn et Paepe, 1968).

1 - Formations fluviatiles de la Meuse et du Rhin: 2 - Zone des sables de couverture : 3 -Zone de transition sablolimoneuse ; 4 - Zone des lœss : 5 - Dépôts holocènes.

Map of the Upper Pleistocene in Northwestern Europe (according to Zagwijn \& Paepe, 1968). 1 - Fluvial deposits of the Meuse and Rhine Rivers ; 2 - Cover Sands Zone; 3 - Sandy-loam Transitional Zone : 4 - Loess Zone : 5 Holocene deposits.

1 Carte disponible gratuitement à l'adresse suivante: ANDRA. Direction de la Communication. Parc de la Croix-Blanche. 1-7, rue Jean. Monnet, 92220 Chátenay-Malabry Cedex. 


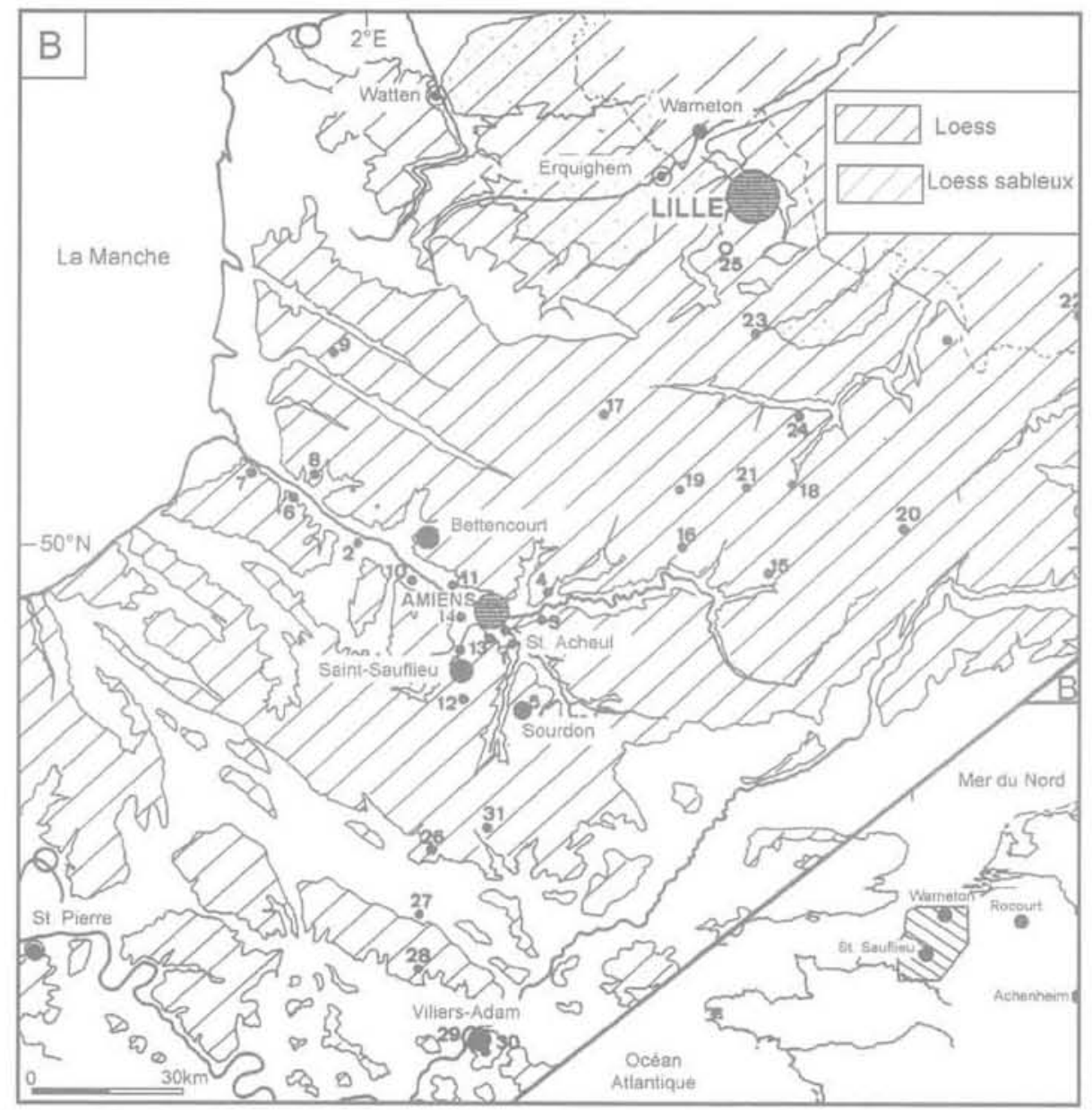

FG.7 Carte d'extension des laess du dernier glaciaire (Weichselien) dans le Nord de la France, localisation des séquences types et de quelques autres points d'observation (d'après Antoine et al., 1998, modifié).

1 -Boves ; 2 -Longpré; 3 - Villers-Bretonneux (Gazoduc) ; 4 -Corbie (Gazoduc) ; 5 - Sourdon ; 6 -Mautort 7 - Boismont ; 8 -Saint-Riquier ; 9 - Gouy-Saint-André ; 10 - Saint-Pierre-à-Gouy ; 11 - Saint-Sauveur ; 12 - Fransures; 13 - Sains-en-Amiénois ; 14 - Plachy-Buyon; 15 - Roisel ; 16 - Combles ; 17 - Beaumetzles-Loges ; 18 - Marcoing : 19 - Bapaume ; 20 - Busigny ; 21 - Hermies ; 22 - Harmignies ; 23 - Corbehem ; 24 - Hamel; 25 - Seclín ; 26 - Beauvais-la-Justice; 27 - Auteuil ; 28 - Lormaison ; 29 - Villiers-Adam ; 30 - Chauvry ; 31 - Saint-Just-en-Chaussée.

Extension map of the last glacial loess cover (Weichselian) in North France, location of type-sequences and of some other observation points (according to Antoine and al., 1998, modified).

tracé du TGV Nord (Antoine, 1991) montrent que la zone Nord-Picardie s'inscrit dans la zone occidentale dans sa partie sud (sud de Bapaume) et dans la zone orientale pour sa partie Nord.

\section{2}

\section{Origine}

En se basant sur la comparaison entre la carte des zones lœessiques et les données minéralogiques (minéraux lourds et argiles), il est possible de localiser les principales zones source des loess weichseliens. Ce travail a permis de distinguer un stock provenant de la Manche centrale. un stock nordique originaire de la mer du Nord, un stock breton (Manche orientale à l'Ouest du Cotentin) et des stocks plus locaux liés à la déflation dans les grandes vallées alluviales (Seine, Oise, Aisne, Marne, Somme...) et pollués par des éléments du substrat (sable, craie gélivée).

D'après ces travaux, l'origine des lœess est donc à rechercher dans la déflation éolienne qui s'exerçait alors à la surface des paléoestuaires émergés de la Seine et de la Somme et des larges plaines alluviales à chenaux en tresses des vallées périglaciaires.
En effet, d'après J.-P. Lautridou (1985), lors du dernier glaciaire (Weichselien), la déflation se serait effectuée sur de grands estuaires situés à 20 ou $30 \mathrm{~m}$ en dessous du niveau actuel de la Manche (Fig. 5), et partiellement conservés lors de la baisse maximum du niveau marin, contemporaine du dernier maximum glaciaire (-140-150 m env.). Dans ce schéma, la sédimentation loessique de la zone Nord-Picardie aurait été alimentée par la déflation à la surface du paléo-estuaire de la Somme, Plus à l'est, ces apports, provenant des estuaires fossiles, se sont combinés à des apports locaux en provenance des vallées (Oise, Marne, Meuse, Rhin). En ce qui concerne les loess de moyenne Belgique, des travaux basés sur les minéraux lourds ont permis de localiser la zone source des loess weichseliens dans le fond de la mer du Nord, alors exondée, au niveau des plaines à chenaux en tresses de l'outwash du glacier scandinave (Juvigné, 1985) (Fig. 2).

Indépendamment du facteur climatique, la sédimentation løessique est donc fondamentalement conditionnée par la présence de zones sources oủ le matériel est disponible pour la déflation éolienne (Fig. 2). Les principales zones de déflations reconnues en Europe du Nord-Ouest sont donc les plaines exondées (paléo-estuaires et paléochenaux de la Manche et de la mer du Nord dans les- 


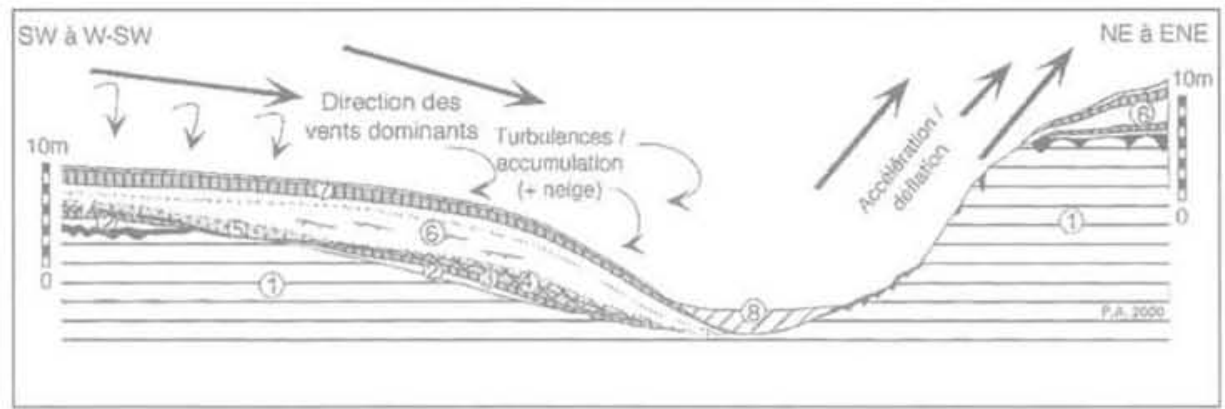

FIG.8 Coupe d'une vallée asymétrique du bassin de la Somme illustrant les relations morphologie/stratigraphie au Pléistocène supérieur.

1 - Craie avec altération argileuse au sommet : 2 - Loess saaliens : 3 - Horizon Bt de sol brun lessivé (interglaciaires) ; 4 - Complexe de sols humifères de Saint-Sauflieu ; 5 -Loess brun à structure feuilletée ; 6-Loss calcaires ; 7 - Colluvions.

Cross section trough an asymetrical valley of the Somme basin showing the relations between morphology and stratigraphy during the Upper Pleistocene.

1 -Chalk with clavey weathering horizon at the top : 2 - Saalian loess: 3 - Bt horizon of brown leached soil (Interglacial): 4 - Saint-Sauffieu humic Soil Complex (Grey-forest solis /steppe soils) ; 5 - Brown loess with foliated structure; 6Typical calcareous loess : 7 - Colluvium.

quelles s'accumulent les sédiments érodés par le réseau hydrographique (Fig. 5), et les grandes vallées alluviales occupées par un systeme de chenaux en tresses pendant les phases pléniglaciaires. Dans ces systèmes fluviatiles typiques des envirornements périglaciaires, les multiples barres sablo-graveleuses peu végétalisées qui séparent les chenaux sont alors le siège d'une déflation éolienne intense.

\section{Morphologies}

Les dépôts lœssiques de l'Europe du Nord-Ouest se présentent sous plusieurs types de morphologies conditionnées par les environnements de dépôt et la présence de pièges sédimentaires :

1) Les lcess de " couverture » se présentent sous la forme d'un manteau d'épaisseur relativement constante (1.5 à 2 m. Fig. 5 , partie supérieure de 1), et se caractérisent par une grande continuité et un faciès très homogène. Ces dépôts correspondent à la phase la plus froide et la plus sèche du Pléniglaciaire weichselien supérieur (1520000 BP env.).

2) Les dépôts lœessiques plus localisés, d'épaisseur variable, et conservés dans des pièges sédimentaires: - les loess des versants abrités ( sous le vent $n$ ) des vallées asymétriques (Fig. 8). Cette morphologie des dépôts loessiques, extrêmement fréquente dans la Somme et le Nord de la France au début du Weichselien supérieur (20$25000 \mathrm{BP}$ ), est conditionnée par l'orientation des vallées ou vallons par rapport aux vents dominants (Ouest à NordOuest dans la Somme et en Normandie, Nord-Ouest à Nord dans le Nord de la France). Les lœess s'accumulent en effet le long du versant sous le vent, où les turbulences engendrées par la morphologie provoquent le dépôt des particules et où la couverture nivale bien développée favorise leur piégeage. Au contraire, le versant exposé au vent se caractérise par une accélération de la vitesse du vent et une absence de dépôt (déflation);

- le même type de phẻnomène se produit dans le cas de structures pièges plus ponctuelles, mais localement très marquées, comme les talus de terrasses alluviales: Somme, Seine, Rhin (Sommé et al, 1986 : Lautridou, 1985,
Brunnacker, 1986 ; Haesaerts et Dupuis, 1986 ; Antoine, 1989b, 1994), ou encore à la jonction entre une ancienne falaise marine et un niveau de plage fossile comme à Sangatte dans le Pas-de-Calais (Antoine, 1989a). Ces séquences d'extension locale se distinguent souvent par une forte influence du substrat qui se traduit par présence de dépôts soliflués ou ruisselés interstratifiés dans la séquence løessique (sables, granules de craie, cailloutis, coulées boueuses) :

3) Les $\alpha$ dunes $»$ de loess (lcess Greda). Cette morphologie en dunes allongées pluri-kilométriques, essentiellement décrites en Europe centrale (Léger, 1990), a aussi été observée sur la rive droite de la vallée du Rhin dans la région d'Heidelberg (Antoine et al., 2001). Dans ce secteur, l'accumulation lœssique essentiellement représentée par les dépôts du Pléniglaciaire supérieur (30-15 ka) atteint 15 à $20 \mathrm{~m}$ d'épaisseur au centre de structures dunaires allongées de plusieurs kilomètres, d'axe NNW-ESE, séparées par de petits vallons.

\section{4}

\section{Grandes lignes de la séquence du demier cycle (Eemien-Weichselien)}

Les données stratigraphiques, paléopédologiques et palynologiques récentes (Antoine, 1989b, 1991: Antoine et al., 1994, 1998, 2001), couplées aux résultats des êtudes sédimentologiques, des mesures de susceptibilité magnétique et les datations TL/IRSL (Engelmann et al., 1999), débouchent sur une nouvelle représentation de la stratigraphie du dernier cycle dans la France du Nord-Ouest (Fig. 9), et de ses connexions avec les régions avoisinantes (Antoine et al., 1999a, 2001) (Fig. 10).

\section{Début-Glaciaire weichselien ( $1112-68 \mathrm{ka} \mathrm{BP}$ ):}

Après la troncature de l'horizon Bt complexe attribué à l'interglaciaire Eemien le début-weichselien est représenté par un complexe de sols humifères (Fig. 9, nos 16 à 21), quí traduit donc une continentalisation du milieu en deux grandes phases contemporaines d'une baisse importante du niveau marin (supérieure à - $20 \mathrm{~m}$ : Sommé et al., 1994) et d'un changement paléogéographique majeur dans le 


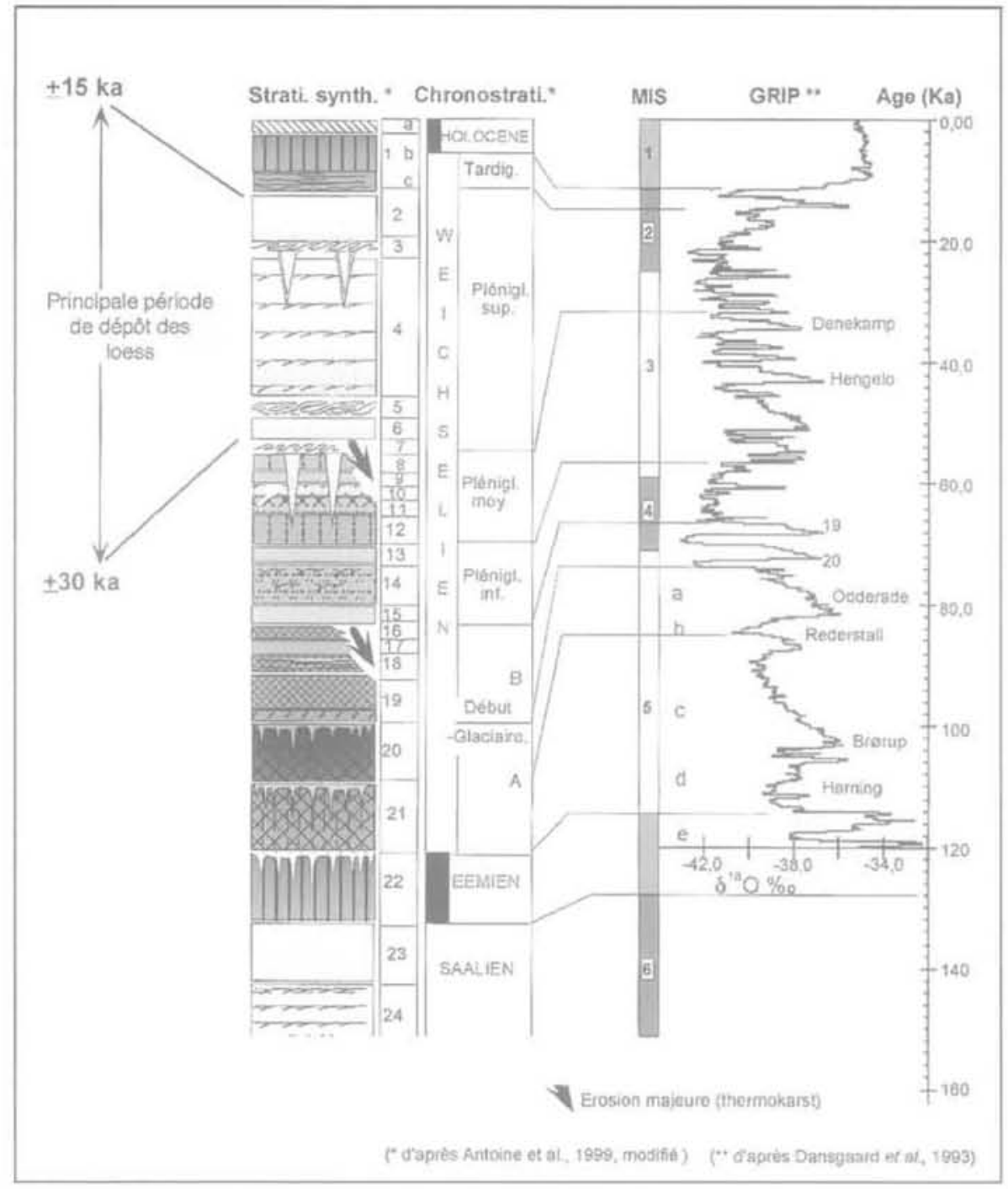

FIG.9 Séquence pédosédimentaire synthétique du Pléistocène supérieur du Nord de la France ; corrélation avec les enregistrements globaux (d'après Antoine et al., 1999, modifíé).

1 - Sol de surface (a: Hz L; b: Hz Bt; $\mathrm{c}: \mathrm{Bt}$ en bandes " horizon à doublets $»): 2$ - Lœss calcaire homogène; 3 -Horizon à langues de Nagelbeek ; 4 - Loess calcaire lité à microfentes ; 5 -Gley de toundra cryoturbé ; 6 - Løess calcaire homogène : 7 - Horizon à langues de gélifluxion/grandes fentes de gel (Ice wedges cast) ; 8 à 12 - Complexe de sols de Saint-Acheull-Villiers-Adam : 8-Sol brun arctique, 9 - Limons sableux lités, 10 - Gley de toundra, 11 - Sol humifêre de type prairie arctique, 12 - Sol brun boréal ; 13 Lœss sableux : 14-Colluvions litées à nodules de sol et cryoturbations syngénétiques ; 15 - Lœss brunâtre; 16 à 20: Complexe de sols de Saint-Sauflieu, 16/18/19 - sols humiferes steppiques, 17 - Loess local non calcaire, 20 - Sol gris forestier; 21 - Sol de Bettencourt (colluvions argileuses/sol gris forestier) ; 22 - Sol brun lessivé glossique tronqué (sol de Rocourt) ; 23 et 24 - Lœss calcaire saalien

Northern France Upper Pleistocene summarised pedosedimentary record; correlation with the global climate records (according to Antoine et al, 1999, modified),

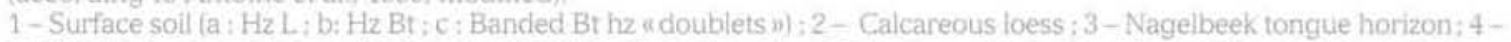
Bedded calcareous loess: 5- Cryoturhated tundra gley : 6 - Calcareous loess : 7 - Tonque horizon/lce wedges: 8 to 12 - Saint-Achetu/Villiers Adam soil complex, 8 - Arctic brown soll, 9 - Stratified sandy loams, 10 - Tundra gley, 11 . Humic soil (arctic meadow) 12- Boreal brown soll ; 13 - Calcareous loess ; 14- Bedded colluvium with frost wedges; 15 Brownish lcess : 16-18-19-Steppe-soils : 17 - Non calcareous local loess ; 20 - Grey forest-soil : 21 - Bettencourt Soll (clavey colluvium / grey forest soill : 22 - Bt horizon of brown leached soil (Rocourt Soil) ; $23-24$ - Calcareous Saalian loess.

domaine Manche-mer du Nord (disparition définitive de l'influence océanique) :

- phase à sols gris forestiers construits sur colluvions, forêt boréale à pins et bouleaux $( \pm 112-72 \mathrm{ka}$, Fig. 9, nos 21 et 20) ;

- sols isohumiques steppiques sur dépôts éoliens ( $\pm 68-72$ ka, Fig. 9, nos 16 à 19$)$.

Pléniglaciaire inférieur ( $\pm 68-55 \mathrm{ka} \mathrm{BP})$ :

- mise en place d'un premier dépôt de loess souvent non calcaire et remaniant des éléments locaux au Pléniglaciaire inférieur (lœss brunâtre) :
- un épisode érosif intense avec mise en place de colluvions litées à nodules de sols, cryoturbations et fentes de gel, qui remanient les niveaux sous-jacents (Fig. 9, $\mathrm{n}^{\circ}$ 14);

- nouvelle génération de lcess souvent hétérogène à granules de craie épars.

Pléniglaciaire moyen $( \pm 55$ - $30 \mathrm{ka} \mathrm{BP})$ :

Développement d'un sol ou plus exactement un complexe de sols (Complexe de Saint-Acheul-VilliersAdam) (Fig. 9, ns 8 à 12). Au cours de cette période, la 


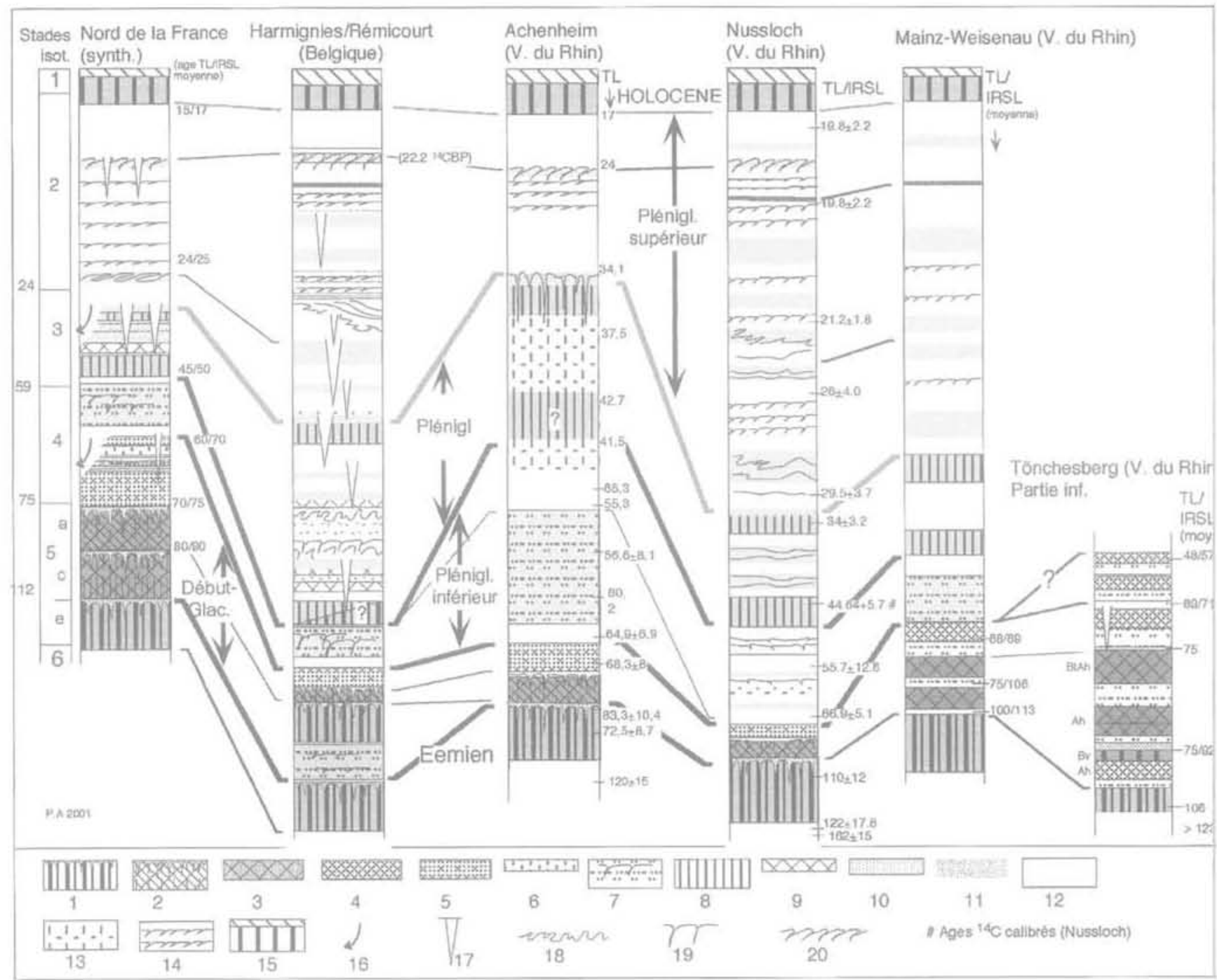

FIG. 10 Corrélation des principales séquences du Pléistocène supérieur du Nord de la France de la Belgique et de la vallée du Rhin (d'après Antoine et al.. 2001).

1 - Horizon Bt tronqué de sol brun lessivé ; 2 - Horizon Bth de sol gris forestier : 3 - Horizons Ah ou Bth de Chernozem (auteurs allemands) ; 4 - Horizon Ah indifférencié ; 5 - Horizon Ah de sol steppique ; 6 Loss local non calcaire ; 7 - Colluvions litées/pellet sands ; 8 - Horizon Bw de sol brun ; 9 - Sol de type prairie arctique ; 10 - Sables ; 11 - Gley de toundra ; 12 - Loss calcaire ; 13 - Loss brun altéré ; 14 - Lœss calcaire finement lité à microfentes de cryodessication : 15- Horizons Bt et Ah du sol de surface: 16 - Érosion majeure ; 17 - Pseudomorphose de grande fente à coin de glace ; 18 - Cryoturbations ; 19 Fentes de gel; 20 - Solifluxion/horizon à langues.

Correlation between main sequences and type sequences from North-Western France. Belgium and Germany (according to Antoine et at, 2001)

1-Truncated Bt hz of Luvisol : 2-Bth hz. of Greyzem on colluvium; 3- Ah or Bth hz of Chernozem (German authors): 4 - Undifferentiated Ah horizon (r.steppe soils v?) : 5 - Ah hz./ ( Steppe Soil $x: 6$ - Local non calcareous loess : 7 Bedded colluvial (locally humic) deposits/Pellet sands : 8 - Bw hz of Cambisol : 9- Humic layer/uarctic meadow soil n; 10 - Sands ; 11 - Gelic Glevsol/Tundra glev; 12 - Calcareous loess: 13 - Weathered loss (u brown loess n) ; 14 - Finelv laminated calcareous loess with cryo-dessication microcracks ; 15 - Bt/Ah hz of the surface Luvisol : 16 - Main erosion: 17 - Ice wedge ; 18 -Cryoturbation; 19-Frost cracks ; 20 - Sollifluxion (tongue hz).

sédimentation lœessique est fortement réduite et interrompue par plusieurs phases de pédogenèse de type sol brun boréal à sol brun arctique, souvent représentées par un horizon unique polyphasé (Antoine, 1989b).

\section{Pléniglaciaire supérieur ( \pm 30-15ka BP):}

Après une coupure majeure, marquée par les premiers réseaux de grandes fentes en coin (permafrost). puis par une érosion importante, le Pléniglaciaire supérieur se distingue par le développement des grandes couvertures lœssiques carbonatées qui ont façonné la morphologie actuelle et représentent la partie la plus dilatée de la séquence ( 4 à $6 \mathrm{~m}$ d'épaisseur, Fig. 9, $\mathrm{n}^{\infty} 2$ à 6). Cette séquence se subdivise au maximum en trois grandes unités séparées par des horizons repères périglaciaires (Antoine, 1991), dont le plus constant est l'horizon à langues de Nagelbeek daté aux environs de $22 \mathrm{ka}{ }^{14} \mathrm{C}$ (Haesaerts et al., 1981).

\section{5}

\section{Granulométrie}

D'après les nombreux travaux menés sur ce sujet (Lautridou, 1985 ; Lautridou et al., 1984 ; Jamagne et al.. 


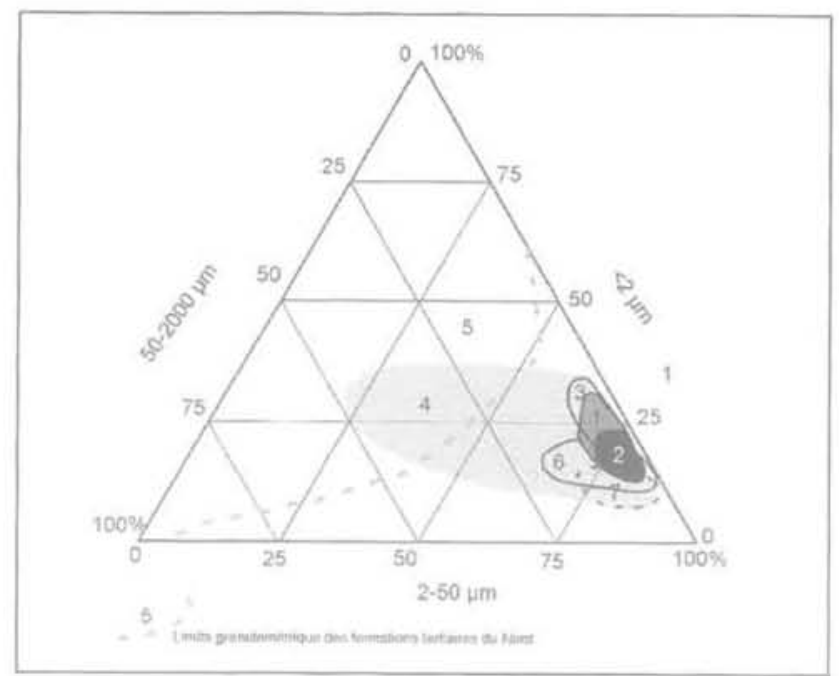

FG. 11 Triangle granulométrique des læss, paléosols, lœss sableux et formations limoneuses litées: comparaison avec les sables tertiaires (d'après Lautridou et al., 1984).

1 - Lœss typiques et paléosols ; 2 - Lœss typiques calcaires: 3 - Loss typiques et paléosols (Hainaut, Ardennes) ; 4 Formations limoneuses litées; 5 - Limite granulométrique des sables tertiaires du Nord; 6 - Limons à doublets de Normandie ; 7 - Loss calcaire typique de Normandie.

Grain-size data triangle for læess, palaeosols. sandy loess and stratified loamy deposits. comparison with the Tertiary sands (according to Lautridou and al., 1984).

1 - Typical loess and palaeosols ; 2 - Typical calcareous loess ; 3 - Typical loess and palaeosols (Hainaut, Ardennes) ; 4 -stratified loamy deposits : 5 - Grain size boundary of the Northern France Tertiary sands ; 6 - Norman " Doublets loess 3: 7 - Norman typical calcareous loess.

1981), et les nouvelles données acquises en séquence continue (Antoine et al., 2001, Fig. 13), les principales caractéristiques granulométriques des loess du NordOuest Européen et plus particulièrement du Nord de la France sont les suivantes (Figs. 11, 12 et 13) :

- nette dominance de la fraction limoneuse $(2-50 \mu \mathrm{m})$. et plus précisément de la fraction 20-50 $\mu \mathrm{m}$ (limons grossiers), qui représente généralement entre 50 et $65 \%$ du sédiment total (Figs. 12 et 13). Néanmoins il faut tenir compte du gradient granulométrique lié à la distance par rapport à la zone source (augmentation progressive de la fraction fine et passage des loess sableux aux loess typiques quand on s'éloigne de la zone source, Fig. 6) ;

- la médiane se situe vers 25-30 $\mu \mathrm{m}$ en France et en Belgique:

- dans les loess typiques (non sableux et non argileux), le rapport limon grossier (20 à $50 \mu \mathrm{m}$ ) sur limon fin $(2$ à $20 \mu \mathrm{m})$ est supérieur à 2,2 dans le Nord de la France ;

- la courbe granulométrique cumulative du lœss typique (Fig. 12), est une sigmoïde asymétrique dont la branche supérieure (sables) est très limitée par rapport à la branche inférieure (limons très fins, argile) en raison du faible pourcentage de sable $(200-200 \mu \mathrm{m}: 0,5$ à $1,5 \%$, localement : $2 \%$ max.) ;

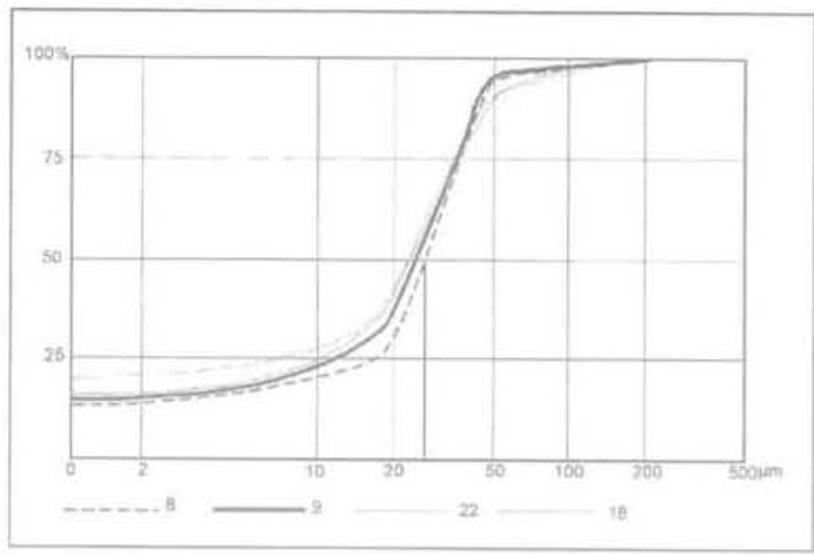

FiG is Exemple de courbes granulométriques cumulatives de lœess de Normandie (SaintRomain) (d'après Lautridou, 1985).

8 - Limon à doublets ; 9 - Lœss brunâtre ; 18 et 22 - Lœess calcaire lité.

Examples of grain-sizes curves of some Norman loess (Saint-Romain) (accorcing to Lautridou, 1985)

8 - Doublets loess : : 9 - Brownish lœess ; 18 and 22 -Laminated calcareous less.

- la branche inférieure montre une faible inclinaison qui traduit un fort pourcentage d'argile fine $(<1 \mu \mathrm{m})$ par rapport à l'argile totale $(<2 \mu \mathrm{m})$. D'une manière générale le rapport d'argile fine sur argile totale dépasse largement $50 \%$; il est cependant plus faible dans les lœss carbonatés (Jamagne et al., 1981), où les particules d'argile fine forment des agrégats avec les particules de carbonates de calcium. Enfin, en ce qui concerne la teneur en argile, on observe une zonation générale matérialisée par un enrichissement en argile des lœess vers le Nord et le Centre est du Bassin parisien (Jamagne et al., 1981).

Par rapport aux loss, les sables éoliens (sables de couverture de la zone belgo-néerlandaise) montrent aussi un très bon classement avec une médiane comprise entre 150 et $300 \mu \mathrm{m}$ (Vandenberghe et Gullentops, 1977). Dans la zone de transition des Flandres, les faciès se chargent progressivement en limon et l'on obtient des compositions intermédiaires ou bimodales (faciès sablo-limoneux de transition).

\section{6}

\section{Minéralogie}

Du point de vue minéralogique, les loess de l'Europe du Nord-Ouest, comme l'ensemble des lœess typiques, se caractérisent par une nette prédominance du quartz dans les fractions sableuses et limoneuses (60 à $70 \%$ en moyenne, Pécsi, 1990). Les autres minéraux sont représentés en proportion décroissante par du feldspath, des micas (muscovite en quantité très variable, biotite), des minéraux lourds (épidote, amphibole, grenat) un peu de glauconie et des argiles (smectite, vermiculite, illite). Dans le faciès typique, la fraction carbonatée est très variable, inférieure à $12 \%$ dans le Nord, la Normandie et la campagne de Caen, elle atteint 15 à $25 \%$ en Picardie et dans le Centre-Est du Bassin parisien (Lautridou, 1985) et dans la vallée du Rhin. 


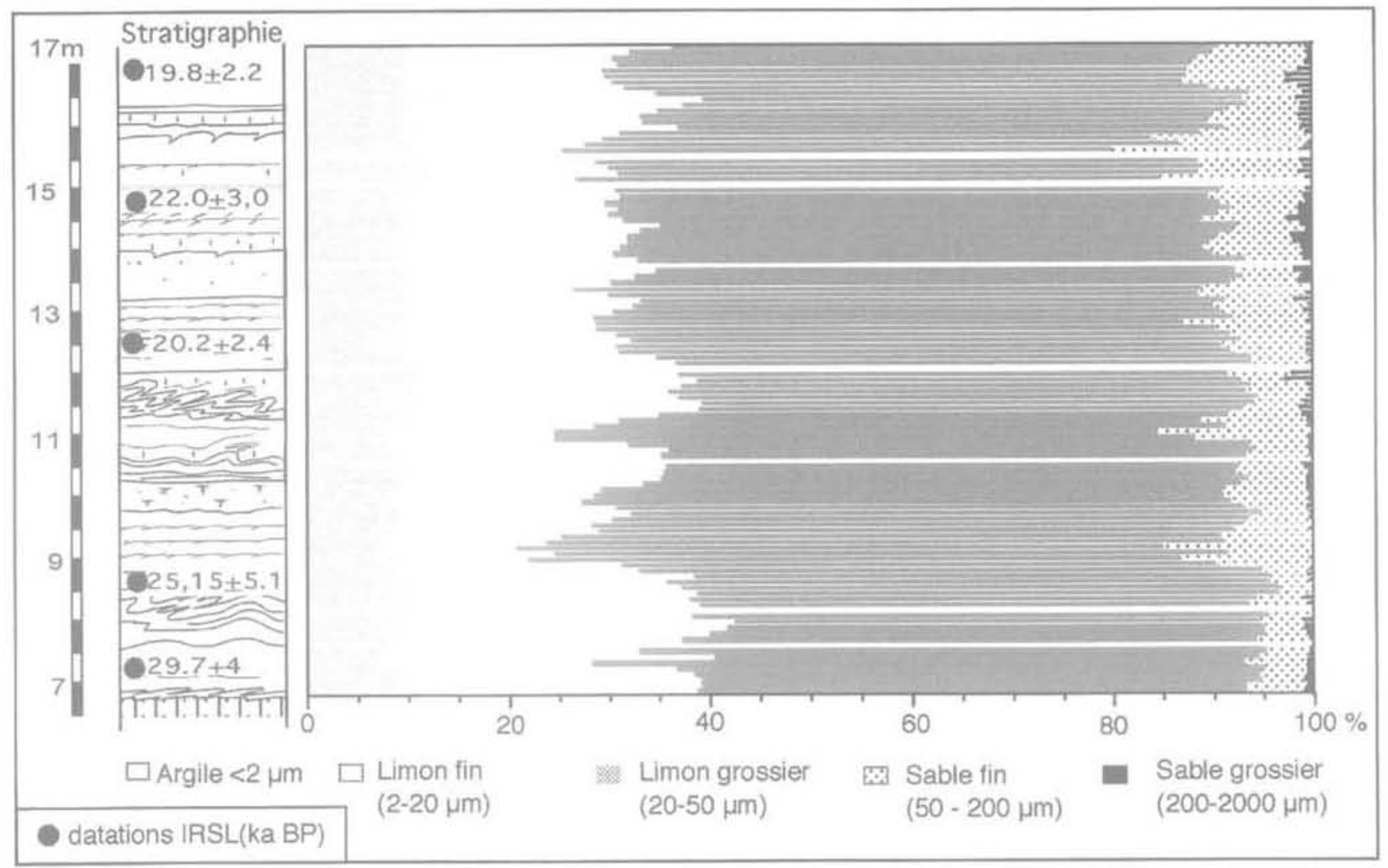

FG. 13 Granulométrie en continu (1 éch/ $10 \mathrm{~cm}$ ) des lœss calcaires du Pléniglaciaire supérieur de la vallée du Rhín (Nussloch-Heidelberg).

Continuous grain-size data (1 sample/10 cm) in the Upper Pleniglacial calcareous loess of the Rhine Valfey (NusslochHeidelberg).

Comme l'ont montré les expérimentations menées au Centre de géomorphologie du CNRS de Caen, la fraction carbonatée sédimentaire des loess (carbonates primaires) est produite par la gélifraction des calcaires du substrat (Lautridou, 1985). Ces carbonates détritiques, générés par la gélifraction des calcaires sur les versants sont entrainés dans les plaines alluviales par les processus de ruissellement et de soliftuxion, puis transitent par le réseau hydrographique en direction des paléoestuaires où ils seront ultérieurement soumis à la déflation. Parallèlement à cette production par gélifraction, une fraction de carbonates primaires est engendrée par l'abrasion des éléments calcaires (blocs galets, granules) en contexte fluviatile dans les plaines alluviales.

L'association de minéraux lourds à épidote, amphibole et grenat, dominante en Normandie, diminue progressivement vers l'Est en faveur des minéraux lourds ubiquistes (minéraux que l'on trouve dans tous les lœess comme le zircon, la tourmaline et le rutile). Par ailleurs, les assemblages de minéraux lourds des lœss weichseliens et saaliens supérieurs (épidote, amphibole, grenat) sont nettement différents de ceux des dépôts antérieurs dominés par les ubiquistes (Balescu, 1986)

$\mathrm{La}$ fraction argileuse inférieure à deux microns est dominée par des argiles mal cristallisées et interstratifiées. Excepté dans le bocage Normand, la vermiculite et la smectite dominent la kaolinite et l'illite. Enfin, le chlorite, présente d'une manière très irrégulière, apparait surtout dans les lœss de l'Ouest de la Picardie et du Bocage normand où elle caractérise les lœss du Saalien supérieur et du Weichselien (Lautridou, 1985).

\section{Microstructure}

Une simple accumulation de poussières éoliennes à la suite de la baisse de la dynamique éolienne (obstacle, zone sous le vent, végétation) ne permet pas d'expliquer la structure particulière du loess typique ( Loess is not just the accumulation of dust », Pécsi, 1990). Cette structure, la fabric des auteurs anglais, se caractérise par une certaine cohésion du matériau et une forte proportion de vides que l'on n'observe pas dans un simple dépôt de poussières éoliennes (Pécsi, 1990; Derbyshire, 1984, Derbyshire et Mellors, 1986). Cette différence est due à lintervention de processus d'altération et de redistribution des carbonates dénommée " lossification $₫$ (Lozec, 1964; Kukla, 1977; Liu et al., 1985).

Au cours de ce processus syngénétique, qui apparait dans les environnements froids arides ou semi-désertiques, les carbonates primaires (calcite, dolomite) sont partiellement altérés puis reprécipités sous la forme de ciments carbonatés. Ces ciments carbonatés participent à la consolidation du matériau et à l'acquisition du faciès loss typique. Les carbonates secondaires peuvent aussi avoir pour origine la dissolution de coquilles de mollusques ou encore la combinaison des ions calcium résultant de l'altération des feldspaths avec le $\mathrm{CO}_{2}$ formé dans les sols (Pécsi, 1990).

Les études basées sur l'analyse microstructurale au microscope électronique à balayage (MEB) (Fig. 14), ont montré que la structure originelle des lcess typiques pouvait se définir par les caractéristiques suivantes (Derbyshire, 1984; Wang et al., 1984; Derbyshire et Mellors, 1986): 


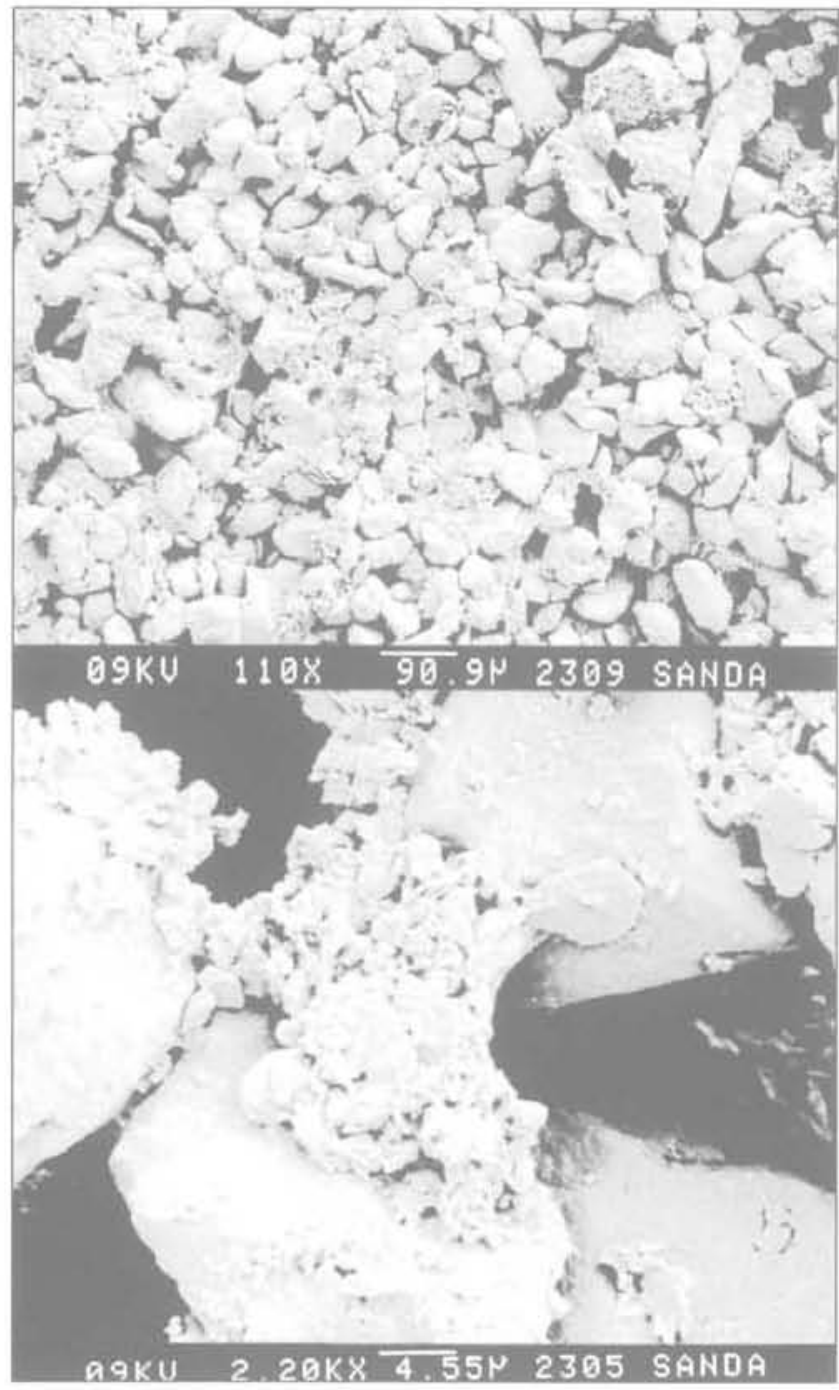

FIG. 14 Microstructure d'un loess typique calcaire au MEB avec micro-agrégats syngnétiques en place (photo du bas) (Longpré, Somme, d'après Balescu, 1988) (photos originales transmises par E. Derbyshire, University of London).

Microfabric of a tvpical calcareous loess under SEM microscope, with undisturbed microaggregates (lower photo) (Longpré. Somme, according to Balescu, 1988) (original photo provided by $\mathrm{E}$. Derbyshire, University of London).

- absence d'orientation préférentielle des grains silteux du squelette (structure isotrope) ;

- forte proportion de vides ( 40 à $60 \%$ ) :

- présence de carbonates secondaires précipités au contact des grains, dans les biopores (racines) et sur les ponts argileux ;

- présence de ponts d'argile qui cimentent les grains silteux et renforcent la cohésion du matériau ;

- présence de revètements argileux sur les grains silteux:

- présence d'agrégats de particules argileuses (buttresses) de forme arrondie et de la taille des silts (10$15 \mu \mathrm{m})$. Ces agrégats syngénétiques sont diagnostiques des lœess typiques en place et participent à leur stabilité. Leur destruction sous l'effet d'une saturation en eau a pour conséquence un effondrement du løess (Derbyshire, 1984).

\section{Processus postdépositionnels}

Cette structure originelle poreuse et isotrope, qui caractérise les loess typiques, peut ètre altérée par des processus postdépositionnels tels que la compaction, la décalcification, la migration ou l'illuviation d'argile, les remaniements (ruissellement, gélifluxion), ou encore les processus liés au gel-dégel. La mise en évidence de ces différents processus est importante car ils modifient les caractéristiques géotechniques en renforçant les ponts intergranulaires et interagrégats (pédogenèse) ou au contraire en les brisant (ruissellement, gel-dégel, hydroconsolidation).

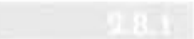

\section{Processus sédimentaires}

Le processus sédimentaire non éolien le plus fréquent dans les séquences lœssiques est le ruissellement. Ce processus détruit la structure originelle isotrope des lcess en introduisant un litage cui se manifeste le plus souvent par l'incorporation de séciments plus grossiers (lits de sable, granules de craie). Ces caractéristiques permettent de distinguer aisément les loess remaniés par ruissellement sur le terrain (litages irréguliers, microchenaux), et lors d'analyses en lames minces (micromorphologie) ou au MEB (apparition de microlaminations, de surfaces d'érosion et de granoclassements, destruction des agrégats, destruction des ponts argileux).

Du point de vue géotechnique, le remaniement des lœess, en réduisant leur porosité, leur confère une plus forte densité et induit une diminution de l'effondrabilité. Par ailleurs, dans certains dépôts lœessiques comme les løess calcaires du Pléniglaciaire de la Normandie et du Nord-Picardie, on observe généralement des faciès finement lités (lcess calcaires lités à microfentes, Figs. 8, 9 et 10), Ces dépôts se caractérisent par l'alternance de niveaux loessiques en place (lits ou lamines de 1 à \pm $10 \mathrm{~mm}$ ), et de lits apparemment plus compacts de lœess remaniés, de coloration plus sombre (brun à grisâtre), quí incorporent localement des granules de craie. Cette structure est souvent interprétée comme la conséquence de la fonte saisonnière de la couverture neigeuse en milieu loessique et rapportée à une dynamique nivéo-éolienne (Lautridou et Sommé, 1974; Mücher et al., 1981; Vreeken et Mücher, 1981 ; Dijkmans, 1990).

Cependant, la présence d'un litage fin millimétrique, caractéristique des lœss du Pléniglaciaire supérieur entre 20 et 25000 BP n'implique pas obligatoirement de phase de transit des particules par ruissellement. En effet, d'après les observations récentes effectuẻes dans la partie sommitale de grandes dunes de loess de la rive droite de la vallée du Rhin (Nussloch-Heidelberg. Fig. 10), cette structure finement laminée apparaît formée par l'accumulation d'une multitude de lamines loessiques en place, de granulométrie variable, correspondant à des épisodes de type tempête de poussière (Antoine et al., 2001).

\section{Fiag}

\section{Processus périglaciaires}

Les traces de l'impact des phénomènes périglaciaires sont particulièrement abondantes dans les 


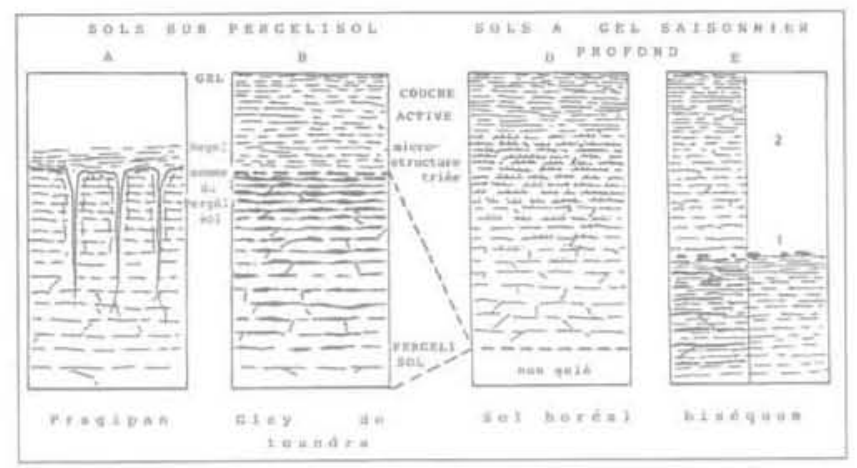

FIG. 15 Structures de ségrégation de glace dans les sols limoneux (d'après Van Vliet-Lanoë, 1987).

Lee segregation structures in loamy soils (according to Van Vliet-Lanôe, 1987).

séquences lœssiques de l'Europe du Nord-Ouest (Pissart, 1987 ; Van Vliet-Lanoë, 1987 ; Huijzer, 1993; Vandenberghe et Pissart, 1993). Leur prise en compte est importante du point de vue géotechnique, car ils provoquent des modifications majeures du comportement mécanique des loess. Les principaux phénomènes liés à l'action du gel dans les loess et les paléosols sont les suivants :

- Les structures de gel-dégel et de gel saisonnier profond (Fig. 15)

Dans les sols limoneux, un gel lent et progressif engendre la formation de lentilles de glace de ségrégation (Pissart, 1987; Van Vliet-Lanoë, 1990). Le développement de ces lentilles de glace, sub-parallèles aux isothermes du sol, entraine la formation d'agrégats polyédriques. La succession de nombreuses phases de gel-dégel s'accompagne d'une compaction et se traduit par l'apparition de structures polyédriques à lamellaires (Pissart, 1987 ; Van Vliet-Lanoë, 1987). Cette compaction mécanique et physique provoque une consolidation des sols limoneux assimilable en géotechnique à une forme de surconsolidation. En effet, les aqréqats subissent une ultra-dessication, et une très forte compaction lors de la croissance des lentilles de glace (réduction de la porosité de drainage). Parallèlement la pression engendre un lissage de la surface des agrégats (stress cutans, Brewer, 1964). Ces phẻnomènes ont pour consèquence une très grande stabilité des agrégats qui pourront ètre remaniés par gélifluxion ou par ruissellement sans être détruits. Dans les sols à gel saisonnier profond ( 1.5 à $2 \mathrm{~m}$ ), la structure s'épaissit progressivement en profondeur $(0,5$ à $\pm 2 \mathrm{~cm})$, en liaison avec l'amortissement de l'onde de gel. En revanche, dans les permafrosts, le sommet de la couche perpétuellement gelée se marque par une discontinuité brutale de la structure lamellaire.

Dans le cas d'un gel-dégel profond, les agrégats prennent progressivement une forme lenticulaire au cours des alternances gel-dégel. Parallèlement, leur sommet se couvre d'une coiffe de matériel fin (silts et argiles) légèrement stratifiée puis d'un revètement granoclassé de grains libres d'un à $10 \mathrm{~mm}$ d'épaisseur (Brewer, 1976). Cette structure particulièrement typique du gel-dégel profond correspond à la " banded fabric $x$ de J. Dumanski (1964).

- Cryoreptation (frost creep), gélifluxion et solifluxion

Ces processus, très fréquents dans les séries lœessiques, correspondent à différents stades de déstabili- sation de la structure de ségrégation de glace sous l'effet d'une pente et (ou) de l'augmentation de la surcharge hydrostatique.

La cryoreptation se traduit par l'affaissement du sédiment au fur et à mesure de son dégel sur pente (Washburn, 1967; Van Vliet-Lanoë, 1987). En présence d'une pente mème très faible $\left(1^{\circ}\right)$, les agrégats formés par le gel-dégel subissent une rotation au moment du décel et l'ensemble du sédiment est alors affecté par un mouvement d'écoulement lent (fauchage). Si la pente augmente ou si le processus s'accélère, les agrégats glissent progressivement les uns sur les autres et s'arrondissent par rotation (cryoreptation accélérée, Van Vliet-Lanoè, 1987). Le stade ultime de cette dynamique est représenté par la gélifluxion qui se traduit par la mise en place de véritables coulées boueuses.

- Cryoturbations et pseudomorphoses de coins de glace

Les cryoturbations et les pseudomorphoses de coins de glace apparaissent à plusieurs niveaux dans la séquence lœessique de l'Europe du Nord-Ouest et constituent des horizons repères pour la corrélation des différents profils (Lautridou et Sommé, 1974; Haesaerts, 1985) (Figs, 10 et 16). A la suite des travaux de A. Pissart (1987), de B. Van Vliet-Lanoë (1987), les cryoturbations sont interprétées comme le résultat d'un gonflement cryogénique différentiel (différence de dilatation des matériaux en fonction de leur gélivité). En effet, dans le cas de gélivités contrastées (ex.: limon/sable), les expériences menèes au Centre de géomorphologie de Caen montrent clairement la formation de structures en gouttes ou en poires lorsque la pression engendrée par ce phénomène est bloquée en surface lors du regel. Dans le cas d'un milieu mal drainé à nappe affleurante, le gonflement cryogénique, bloqué par la rigidification superficielle lors de l'engel provoque une déformation en goutte vers le bas. Par contre en milieu drainé, le gonflement cryogénique peut s'exprimer vers la surface et donne naissance à des formes en relief (ostioles, sols à buttes).

Les coins de glace sont des formes dérivant des fentes de contraction thermique, ils se forment progressivement par la succession de phases de fissuration du permafrost par contraction thermique en hiver et de comblement par de la glace de regel résultant de la fonte de la couverture neigeuse au printemps (French, 1996: Murton et French, 1992,1993a, b : Péwé, 1962: Pissart, 1987 ; French et Godzick, 1988 ; Harry et Godzick, 1988). La formation de ces structures témoigne de la présence d'un permafrost, d'une température moyenne annuelle inférieure à $-8{ }^{\circ} \mathrm{C}$ et de l'absence de couverture neigeuse épaisse

A la suite de la dégradation du coin de glace, la structure est le plus souvent fossilisée par les apports loessiques (pseudomorphose de coin de glace, Fig. 16). Ces structures qui atteignent couramment $1 \mathrm{~m}$ d'ouverture pour $2 \mathrm{~m}$ de profondeur dans les loess du Nord-Picardie s'organisent en réseau polygonal à maille décamétrique.

\section{Processus pédologiques}

Comme tout dépôt sédimentaire, les løess sont susceptibles d'être affectés par une pédogenèse. Ce phé- 


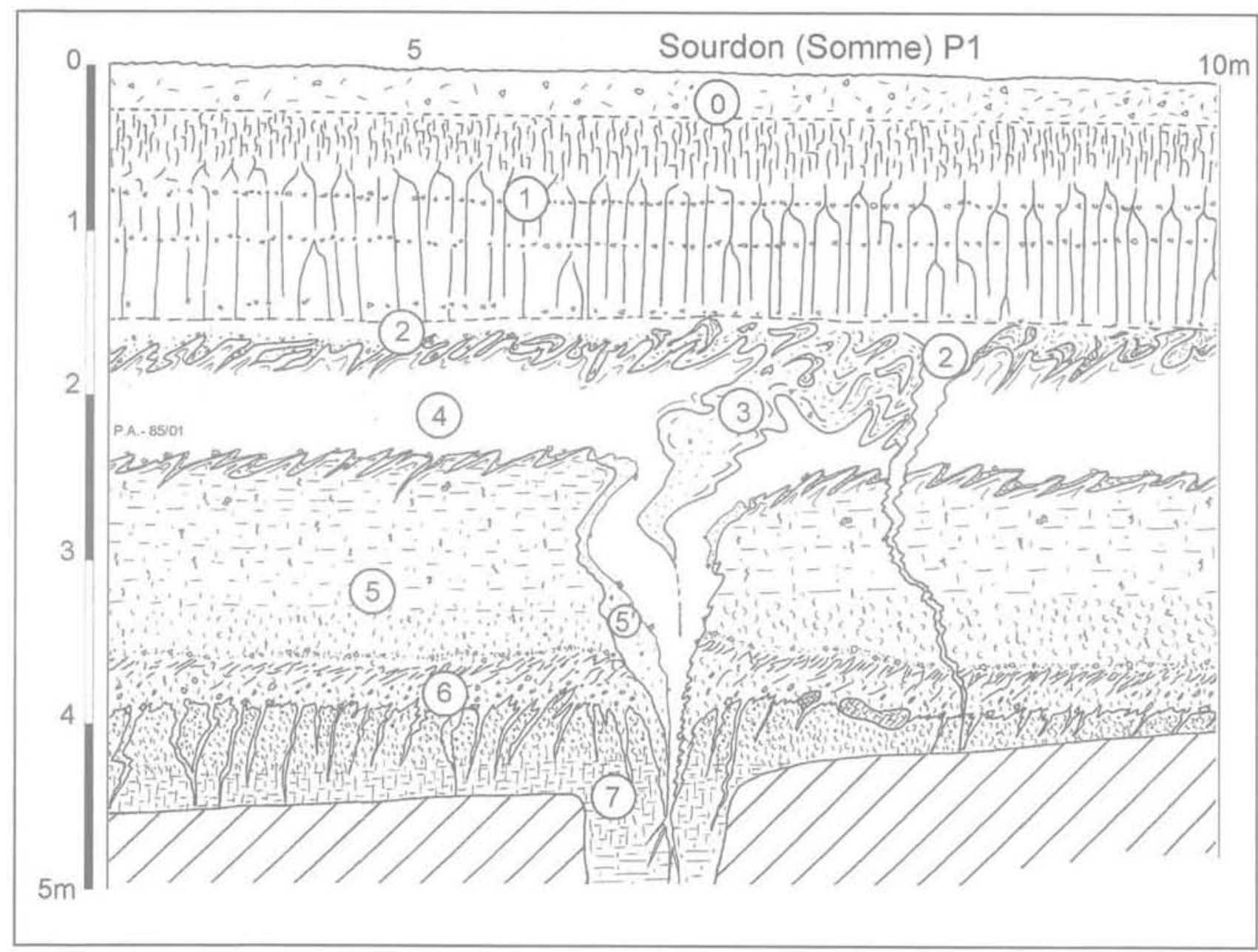

fIG. 16 Levé partiel de la coupe de Sourdon (Somme, localisation: Fig. 7), montrant une pseudomorphose de grande fente à coin de glace et des horizons à langues (d'après Antoine, 1990, modifié).

0 - Horizon humifère de surface ; 1 - Horizon Bt du sol brun lessivé de surface ; 2 - Loess calcaire homogène ; 3 - Horizon grisâtre à langues de gélifluxion ; 4-Lœss calcaire homogène ; 5 - Lœss brun à structure feuilletée : 5' - Lœss grisâtre géliflué ; 6 - Horizon humifère bioturbé ; 7 - Horizon Bt complexe tronqué (dernier interglaciaire).

Partial drawing of the Sourdion section (Somme, location: Fig. 7) showing ice wedges cast and tongue horizons (according to Antoine, 1990, modified):

0 - Humic horizon of the surface soil ; 1 - Bt horizon of the surface brown leached soil ; 2 - Calcareous loess ; 3 - Grevish loam with gelifluxion tongues; 4 - Calcareous loess ; 5 - Brown loess with foliated structure : 5 ' - Geliftucted greyish loam ; 6-Bioturbated humic horizon ; 7 - Complex Bt horizon of a brown leached soil (Last Interglacial).

nomène modifie d'ailleurs fortement les caractéristiques initiales des lcess (baisse de la porosité, augmentation de la résistance à la compaction...). Par exemple, des mesures au scissomètre portable, effectuées sur une séquence de loess à proximité du tracé du TGV Nord, ont montré une augmentation très sensible de la résistance mécanique au cisaillement entre un loess calcaire $(250 \mathrm{kPa})$ et le même lœess décarbonaté et pédogénéisé $(650 \mathrm{kPa})$.

D'une manière simplifiée, dans les lœess calcaires de la France du Nord, il est possible de distinguer deux grands ensembles de pédogenèse : les pédogenèses caractérísées par une illuviation d’argile ( \pm humifère). et généralement précédées par une décarbonatation totale (sol brun lessivé, sols gris forestier), et les pédogenèses sans illuviation d'argile (sol brun arctique/boréal, sol humifère steppique/sol de « prairie arctique \#, gley de toundra).

\section{- Pédogenèses avec illuviation d'argile (lessivage)}

La décarbonatation représente le stade initial de l'altération des lœess calcaires au cours du processus de pédogenèse de type sol lessivé ou sol brun lessivé (Duchaufour, 1984; Van Vliet-Lanoë, 1987) (Fig. 17). Ce phénomène intervient dans un contexte de transition, en début d'amélioration climatique, en liaison avec la fonte du pergélisol. Pour les lœess weichseliens, la décarbonatation đébute à la fin du Pléniglaciaire vers 16000 BP environ (Van Vliet-Lanoë et al., 1992). Elle se manifeste tout d'abord par la formation de niveaux à doublets (succession de bandes à texture plus fine de 0,5 à $2 \mathrm{~cm}$ d'épaisseur dans les derniers lœss). L'origine de ces structures pédologiques typiques des løess sousjacents à un horizon d'accumulation des argiles (horizon $\mathrm{Bt}$ ) est reliée à une illuviation précoce (dépôt d'argiles) sur des surfaces de discontinuités générées par le gel.

La deuxième phase d'illuviation d'argile est liée à la dégradation définitive du pergélisol au cours de l'interstade de Bølling vers 13000 BP (Fig. 17). Ce processus entraîne une remobilisation des argiles présentes dans le matériau loessique dans l'ensemble de la matrice du sédiment et débouche sur la formation de l'horizon Bt du sol de surface. Du point de vue géotechnique, cet horizon 


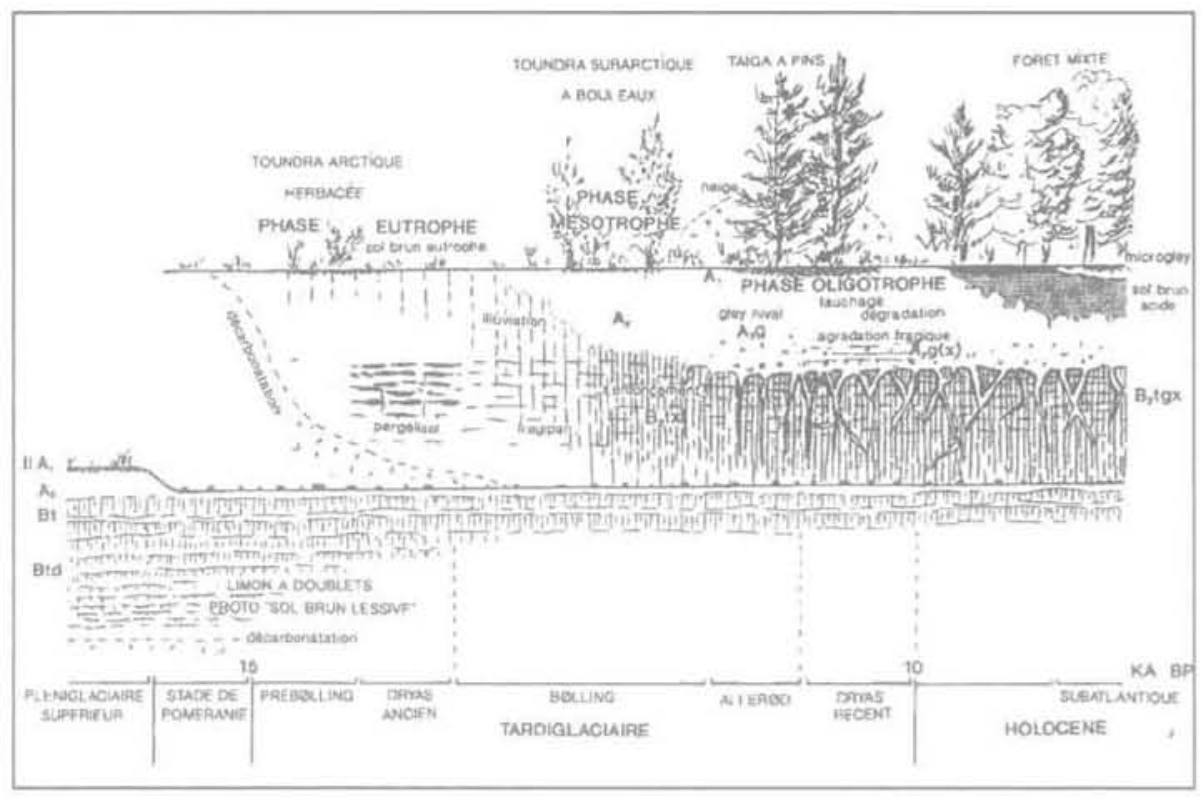

FIG. 17. Schéma de l'évolution pédologique du sol de surface en milieu loessique depuis 16000 ans environ (d'après Van Vliet-Lanöe, et al., 1992).

Summarised diagram of the pedological evolution of the surface soil in loessic environment since $16000 \mathrm{BP}$ (according to Van Vliet-Lanoë and al., 1992).

est fondamental car il isole et protège les dépôts lœessiques carbonatés sous-jacents du ruissellement, des trop fortes variations d’humidité et du gel-dégel qui peuvent entraîner des dégradations irréversibles de la structure des loess au niveau d'un chantier (formation de ravines de ruissellement, effondrements). En cas de décapage en profondeur d'un chantier ( 1 à $2 \mathrm{~m}$ suivant les cas), on retrouve ainsi en surface une couche de loess non altéré et non saturé, qui conserve sa forte porosité et son potentiel d'effondrement.

Dans la séquence des lœess weichseliens du NordPicardie, on retrouve des pédogenèses de même type à la base de la séquence, au niveau du demier interglaciaire (Eemien : 112 à 128000 BP). Cependant, par rapport à 1 horizon argileux de surface, l'horizon Bt du sol brun lessivé contemporain de la fin de l'avant-dernier glaciaire et du dernier interglaciaire présente des différences importantes liées à la surimposition de plusieurs phases de pédogenèse et de gel saisonnier profond, contemporaines du début du Weichselien (horizon Bt polyphasé, Fig. 9, n 22). Cette succession se traduit par une plus forte compaction, une teneur en argile plus importante (25 à $30 \%$ ), et une structure polyédrique résultant du gel saisonnier profond $(-1,5$ à $-2 \mathrm{~m}$ ) contemporain des phases froides du début Weichselien ( \pm 75-112000 BP).

La base des séquences løssiques weichseliennes de l'Europe du Nord-Ouest montre très fréquemment des sols humifères argileux de type gris-forestiers (Driessen et Dudal, 1991; Gerasimova et al., 1996), formés sur des colluvions limoneuses issues de l'érosion du sol interglaciaire. Ces sols se distinguent nettement des sols bruns lessivés par une coloration brun noir, une forte bioturbation, et des illuviations argilo-humiques à silto-humiques stratifiées épaisses et une forte structuration par le gel. Ils correspondent à un contexte climatique continental frais (précipitations $<600 \mathrm{~mm} / \mathrm{an}$ ) et sont attribuables à la première phase du Début-Glaciaire (Antoine et al., 1994, 1998). Du point de vue mécanique, ils se distinguent par une forte compacité et une résistance mécanique élevée en liaison avec leur forte teneur en argile (25 à $30 \%$ ). Par contre la présence d'une forte structure de gel saisonnier (structure prismatique à lamellaire centimétrique à enduits de silts blancs), qui leur confère un débit en blocs centimétriques, introduit de nombreuses discontinuités pouvant favoriser une infiltration rapide entre les agrégats polyédriques hypercompactés.

- Pédogenèses sans illuviations argileuses, sols isohumiques et gleys de toundra

Dans les séquences lœssiques de l'Europe de I'Ouest le Pléniglaciaire moyen ou Interpléniglaciaire (Haesaerts, 1985) (30-50 000 BP), se caractérise par le développement d'un sol ou d'un complexe de sols bruns de type boréal, qui traduit une amélioration climatique relative (toundra à bouleaux), par rapport aux conditions qui régnaient lors du dépôt des lcess. Ce sol, bien visible dans la plupart des sondages effectués sur le tracé du TGV-Nord (Antoine, 1991), se matérialise par un horizon brun argileux sans illuviation, à forte structure lamellaire et par l'abondance des précipitations carbonatèes à la base (biotubules, pseudomycelium). Sa structure lamellaire fine « feuilletée » est héritée de la phase de ségrégation de glace contemporaine de l'établissement d'un permafrost vers 30000 BP.

Les séquences lœssiques du Nord-Ouest européen se distinguent aussi par la présence de pédogenèses peu évoluées (pédogenèses de milieux froids), sans illuviation d'argile et sans décarbonatation notable :

- les sols humifères sans illuviation d'argiles sont représentés par les sols isohumiques steppiques et les sols de prairie arctique (Van Vliet-Lanoë, 1987). Ces sols peu épais $(0,2$ à $0,5 \mathrm{~m})$, de coloration grisâtre, s'individualisent par une humification homogène et une forte bioturbation (brassage par les insectes et larves d'insectes). Ils apparaissent essentiellement dans la deuxième partie du Début-Glaciaire dans la phase steppique entre 65 et $70000 \mathrm{BP}$ environ (Van Vliet-Lanoë, 1987 ; Antoine et al., 1994, 1998, 1999a) : 
- les gleys de toundra sont fréquents dans les loess de l'Europe du Nord-Ouest essentiellement au cours du Pléniglaciaire supérieur (25-20 000 BP) et souvent associés à des pseudomorphoses de coins de glace et à des cryoturbations (Fig. 9, nos 5 et 7). Ils se distinguent par une déferrification en milieu temporairement hydromorphe en liaison avec un engorgement saisonnier de la couche active du permafrost au cours du dégel estival (Van Vliet-Lanoë, 1987). Les données analytiques montrent que ces horizons se caractérisent par une migration du fer, des carbonates et des argiles vers la base de la couche active. Du point de vue mécanique ils s'individualisent par une plus forte compacité,

\section{4}

\section{Conclusion}

Les lœss sont largement représentés dans l'hémisphère Nord et, plus particulièrement, en Europe aux latitudes tempérées, autour de $50^{\circ} \mathrm{N}$, où ils constituent une formation typique des grandes glaciations quaternaires (sédiment éolien caractéristique des contextes périglaciaires).

D'une manière générale, les lcess ne constituent pas une formation homogène mais s'organisent en séquences lœssiques montrant une succession de cycles de type glaciaire-interglaciaire $( \pm 100000$ ans). Cette cyclicité se manifeste par l'alternance de phases de dépôt de lœess typiques carbonatés lors des phases pléniglaciaires, et de paléosols ou de complexes de paléosols qui traduisent des périodes d'arrêt de la sédimentation, d'altération et de pédogenèse dans un contexte d'amélioration climatique (interglaciaire ou interstadiaire). Ces séquences lœessiques ont depuis longtemps intéressé les quaternaristes qui les ont tout d'abord abordées dans une optique stratigraphique, sédimentologique et génétique. De plus en plus ces séquences font l'objet d'études détaillées dont l'objectif principal est la reconstitution des variations climatiques et des modifications de l'environnement en domaine continental, où elles représentent l'enregistrement le plus complet.

Dans la France septentrionale, les études menées depuis les années 70 se sont nettement développées depuis une dizaine d'années à l'occasion des grands travaux d'aménagement et des opérations de sondage et de fouille liées à l'archéologie préventive. Ces recherches, essentiellement axées sur la séquence du dernier cycle interglaciaire-glaciaire (128-15 ka), débouchent sur un renouvellement des données de terrain qui, associé aux nouvelles datations TL, permet actuellement de définir d'une manière relativement précise la stratigraphie et la morphologie de ces dépôts, et de proposer des corrélations détaillées avec celle des régions limitrophes (Allemagne, Belgique).

Parallèlement, ces travaux nous permettent de mieux comprendre les importantes différences de comportement géotechnique, et notamment d'effondrabilité, qui caractérisent les différentes unités pédo-sédimentaires constituant la série lœssique, en fonction de leur histoire sédimentaire, pédologique et périglaciaire. Enfin, cette connaissance détaillée fournit une base qui permettra à l'avenir d'effectuer une prédiction rapide, à partir de l'analyse des faciès, des problèmes d'effondrabilité pour les chantiers qui se dérouleront dans cette zone løessique du Nord et de l'Est de la France.

\section{Bibliographie}

An Z., Kukla G.L.. Porter C.S., Xiao J. (1991) - is Magnetic susceptibility evidence of monsoon variations on the Lcess Plateau of Central China during the last 130000 years $\%$. Quaternary Research, 36, p. 29-36,

Andersen B.J., Borns H.W. (1997) - The Ice Age World. Scandinavian University Press, Oslo, 208 p.

Antoine P. (1989a) - « Stratigraphie des formations pléistocènes de Sangatte (Pas-de-Calais), d'après les premiers travaux du Tunne sous la Manche 1. Bull. Ass. Fr. Et. Quat., 37, p. 5-17.

Antoine P. $(1989 \mathrm{~b})$ - Les terrasses quaternaires du bassin de la Somme :étude géologique et géomorphologique. Contribution a la connaissance du paléoenvironnement des gisements paléolithiques. Thèse Doct. Univ. Sciences et Techniques de Lille Flandres-Artois, $437 p$

Antoine P. (1991) - « Nouvelles données sur la stratigraphie du Pléistocène supérieur de la France septentrionale, d'après les sondages effectués sur le tracé du TGV-Norci s. Publ. du Centru d'Études et de Recherches préhistoriques de Lille, 3, p. 9-20.

Antoine P. (1994) - "The Somme Valley terrace system (Northern France): A model of river response to quaternary climatic variations since 800000 BP n. Terra-Nova, 6, p. 453-464.

Antoine P. Munaut A.V., Sommé J. (1994) - $\alpha$ Réponse des environnements à l'évolution climatique du Début-Glaciaire weichsélien : données de la France du Nord-Ouest 11. Quaternaire, 5. p. 151-156.

Antoine P., Lautridou J.-P., Sommé J,. et al. (1998) - a Le Quaternaire de la France du Nord-Ouest : limites et corrélations n. Quaternaire, 9 (3), p. 227-241, 1 pl. HT.

Antoine P., Rousseau D.D., Lautridou J.P., Hatté C. (1999a) - $\approx$ Last Interglacial-Glacial climatic cycle in loess-palaeosol successions of North-Western France ». Boreas, 28, p. 551-563.

Antoine (ss la dir. de) (1999b) - "Extension des loess et sables éoliens à $18 \pm 2 \mathrm{Ka}$ en France ». Dans La France pendant les deux derniers extrêmes climatiques, variabilité naturelle des environnements. INQUA et ANDRA (eds). Carte à $1 / 1000000$ et notice explicative, p. 22-26.
Antoine P., Rousseau D.D., Zöller L., Lang A., Munaut, A.V., Hatte C. Fontugne M. (2001) - a High resolution record of the last Interglacial-glacial cycle in the Nussloch loess-palaeosol sequences, Upper Rhine Area Germany w, Quaternary International, 76-77, p. 211-229.

Balescu S. (1986) - a La minéralogie des lcess du Nord de la France, et la position stratigraphique des paléosols intra-lœessiques i. Supll. Bull. Ass. Fr. Et. Quatern., 26, p. 165-170.

Balescu S. (1988) - Apports de la thermoluminescence á la stratigraphie et à la sédimentologie des laess du Nord-Ouest de I'Europe. Thèse Doct. Scien. Univ. Bruxelles, 2 vol. I: 199 p. ; vol. II : annexes, 144 p.

Beer J., Shen C., Heller F., Liu T., Kubik P.W. (1993) - s "0Be and magnetic susceptibilioty in Chinese loess $»$. Geophysical research letter, 20, p. $57-60$.

Brewer R. (1976) - Fabric and mineral analysis of soils. New York, Krieger.

Brunnacker K. (1986) - « Quaternary stratigraphy in the lower Rhine area and Northern alpine foothills \&. Dans \& Quaternary Glaciations in Northern Hemisphere s, Quaternary Sciences Review, 5, p. 373-379.

Buraczynski J., Butrym J. (1984) - « La datation du profil d'Achenheim (Alsace) à l'aide de la méthode de la thermoluminescence n. Bull. Ass. Fr. et Quat, 4, p. 201-209.

Coude-Gaussens (1987) - is The perisaharan loess: sedimlentological characterisation and paleoclimatic significance $\%$. Geo journal, 15 (2), p. $177-185$

Cui Y.J., Schlosser F., Delage P., Wojnarowicz M., Durand (1995) - a Comportement mécanique des lcess sur le tracé du TGV. Nord n. X ECSMFE, Copenhague, $6 \mathrm{p}$.

Damblon F. Haesaerts P., Van Der Plicht J. (1996) - « New datings and considerations on the chronology of the Upper Palaeolithic sites in the Great Eurasian Plain », Préhistoire Européenne, 9, p. $177-231$

Derbyshire E. (1984) - " Granulometry and fabric of the loess at Jiuzhoutai, Lanzou, People's Republic of China 》. Dans Litho- 
logy and stratigraphy of loess ans paleosols, M. Pécsi (ed.), Geographical Research Institute, Hungarian Academy of Science, Budapest, p. 95-103.

Derbyshire E., Mellors T.W. (1986) - « Loess N. Dans A Hand-Book of engineering geomorphology, P.G. Fookes and P. Vaughan (eds.), Surrey University Press and Blackie. Edinburgh, p. 237 246.

Derbyshire E., Wang E., Jin J.T., Billard A., Egels Y., Jones D.K.C., Kasser M. Muxart T. Owen L.A. (1991) - $\alpha$ Landislides in the Gansu loess of China p. Dans in Loess geomorphological hazards and processes m, S. Okuda, A. Rapp, L. Zhang (eds.), Catena, Suppl. 20, p. 119-145.

Diikmans I.W (1990) - « Niveo-aeolian sedimentation and resulting sedimentary structures; Søndre Strømfjord area, Westert Greenland x. Permafrost and Perialacial Processes, 1, p. 83-86.

Dijkstra I.A. (2000) - Loess slope instability in the Lanzhou region. Netheriands Geographical Studies, Univ, Utrecht, $301 \mathrm{p}$.

Ding Z L. Renb J.Z., Su J.M. Liu T.S. (1998) - u Climatic instability during the Penultimate Glaciation: evidence from the Chinese Loess Deposits ग, PAGES-Newsletter, 6-1, p. 3-4

Driessen P.M. Dudal R. (1991) - The major soils of the wortd. Lecture notes on their geography, formation, properties and use. Driessen P.M.. Dudal R. (eds). Agricultural University Wageningen \& Katholieke Universiteit Leuven, p. 245-248.

Dubois G.. Firtion F. (1936) - « Esquisse de l'extension des limons loessiques en France n. Bull. du service de la carte géol. d'Alsace et de Loraine, III, p. 21-26.

Duchaufour P. (1984)-Pédologie. Masson, 220 p.

Dumanski J.A. (1964) - A micropedological study of eluviated horizons. Master Thesis, Univ. of Saskatchewan.

Engelmann A. Frechen M. Antoine P. (1999) - a Chronostratigraphie frühweichseiselzeitlicher kolluvialer Sedimente vor Bettencourt-Saint-Ouen (Nord-Frankreich) ». Dans Terrestrische Quatärgeologie, Becker-Hauman. Frechen M. (eds Logabook, Koln, p. 12-22.

Eden D.N. (1980) - "The loess of North-East Sussex m. Boreas, 9 (3). p. $165-177$

Fisher T.G. (1996) - « Sand wedges and ventifact palaeoenvironmental indicators in North-west Saskatchewan, Canada, 11 ka to $9.9 \mathrm{ka} \mathrm{BP}$.. Permafrost and Periglacial Processes, 7. p. 391408.

Forman S.L., Bettis E.A., Kemmis T.J, Miller B.B. (1992) - " Chronologic evidence for multiple periods of loess deposition diuring the Late Pleistocene in the Missouri and Missisipi River Valley, United-States: implications for the activity of the Laurenticle Ice Sheet 1. Paleogeogr. Palaeo-clim. Palaeoecol.. 93. p. $71-83$.

Frechen M. (1999) - « Upper Pleistocene lœess stratigraphy in Southern Germany 1. Quaternary Geochronology, 18, p. 243-269.

French H.M. (1996) - The periglacial environment. London, Longman, 2nd edition.

French H.M. Gozdzik?? (1988) - « Pleistocene epigenetic and syngenetic frost fissures, Belchatow, Poland $x$, Canadian Journal of Earth Sciences. 25, p. 2017-2027.

Gerasimova M.I. Gubin S.V. Shoba S.A. (1996) - « Soils of Northern Forest-Steppe. Grey forest-soils 1. Dans Soils of Russia and adjacent countries: Geography and Micromorphology, R. Miedema (ed.), Moscow-Wageningen, p. 123-134

GRIP Members (1993) - " Climate instability during the last interglacial period recorded in the GRIP ice core. Nature 364, p. 203207.

Gullentops F. (1954) - Contribution à la chronologie du Pléistocène et des formes du relief en Belgique. Mém. Inst. Géol. Univ. Louvain, XVIII, p. $125-252$

Guo Z. Liu T. Guiot J., Wu N.. Han J.. Liu J.. Gu Z. (1996) - " High frequency pulses of East Asian monsoon climate in the last two glaciations: link with the North Atlantic n. Climate Dynamics, 12. p. $701-709$

Haesaerts P. (1985) - " Les lcess du Pléistocène supérieur en Belgique : comparaison avec les séquences de l'Europe centrale 1). Bull. Ass. Fr. Et. Quat., 22, p. 105-115.

Haesaerts P., Juvigé E., Kuyl O., Mucher H., Roebroeks W. (1981) - " Compte rendu de l'excursion du 13 juin 1981, en Hesbaye et au Limbourg néerlandais, consacrée à la chronostratigraphie des løess du Pléistocène supérieur \%. Annales de la Société géologique de Belgique, T. 104, p. 223-240.

Haesaerts P., Dupuis C. (1986) - is Contribution à la stratigraphie des nappes alluviales de la Somme et de l'Avre dans la région d'Amiens $v$. Dans $\alpha$ Chronostratigraphie et faciès culturels du Paléolithique inférieur et moyen dans I'Europe du NordOuest 1, Suppl. Bull. Ass. Fr. Et. Quat., 26, p. 171-186
Han J.M., Hus J.J., Paepe R., Vandenberghe R.E., Liu T.S. (1991)a The rock magnetic properties of the Malan and Lishi Formations in the Loess Plateau of China w. Dans Loess, Environment and Global Change, Liu T.S. (ed.), Beijing Science Press China, p. $30-47$.

Harry D.G., Gozdzik J.S. (1988) - « Ice wedges: growth, thaw transformatiuon, and palaeoenvironmental significance 10 . Journal of Quaternary Science, 3. p. 39-55.

Hatté C., Fontugne M., Rousseau D.D., Antoine P., Zöller 1., Tis. néra-Laborde N., Bentaleb I. (1998) - ${ }^{19} \mathrm{C}$ Variations of loess organic matter as a record of the vegetation response to climatic changes during the Weichselian v, Geology, 26 (7), p. 583586

Heim J., Lautridou J.-P., Maucourps J., Puisségur J.-J., Sommé J. Thevenin A. (1982) - " Achenheim : une séquence type des loess clu Pléistocène moven et supérieur 3. Bull. Ass. Fr. Et. Quat.، 19, p. $147-159$

Heller F. Evans M.E. (1995) - "Loess magnetism w. Reviews of Geophysics, 33-2, p. 211-240

Huijzer A.S. (1993) - Cryogenic microfabrics and macrostrtuctures interrelations, processes, and paleoenvironmental sianificance. $\mathrm{PhD}$ Thesis Vrije Universiteit Amsterdam, $245 \mathrm{p}$

Jamagne M., Lautridou J.P., Sommé J. (1981) - u Préliminaire à une synthèse sur les variations sédimentologiques des loess de la France du Nord-Ouest dans leur cadre géographique et paléogéographique ». Bull. Soc. Géol. Fr. 7, XXIII, p. 143-147.

Friondo M. (1990) - 4 Map of the South American plains: its present state 3. Dans Rabassa J (ed.), Quaternary of South America and Antarctic Peninsula, vol. 6. Rotterdam, Balkena, p. 297-308.

Jiamao H.. Hus J.J. Paepe R., Vandenberghe R.E., LiU T. (1991) "The rock magnetic properties of the malan and Lishi formations in the Loess Plateau of China m. In : Loess Environment and Global Change, Liu Tungsheng (ed.), Beijing. China. Science Press, p. 30-47.

Juvigné E. (1985) - $\alpha$ The use of heavy mineral suites for loess stratigraphy w. Geologie en Mijnbouw, 64, p. 333-336.

Juvigne H. Wintle A.G. (1988) - "A New chronostratigraphy of the Late Weichselian Loess Units in Middle Europe based on Thermoluminescence Dating y. Eiszeitalter u. Gegenwart, 38, p. 94-105.

Kröhling D.M. (1999) - Sedimentological maps of the typical moessic units in North Pampa, Argentina ग. Quaternary International, 62, p. 49-55.

Kukla G.J. (1977) - « Pleistocene Land-Sea Correlations, IEurope n. Earth Sciences Reviews, 13, p. 307-374.

Kukia G.) (1987) - « Loess stratigraphy in central China n. Quaternary Science reviews, 6, p. 191-219.

Kukla G.J. An Z. (1989) - « Loess stratigraphy in central China ". Paleogeography, Paleoclimatolocy, Paleoecology, 72, p. 203, 225

Kukia G.J. (1987) - "Correlation of Chinese, European and American loess series with deap-sea sediments n. Dans Aspects of loess Research. Liu Tungsheng (ed.), Beijing. China Ocean Press, p, $27-38$.

Kukla G.J., An Zisheng S., Merilce J.L. Gavin J., Xiao J.L. (1990) ". Magnetic susceptibility record of Chinese loess 1. Transactions of the Royal Society of Edinburgh: Earth Sciences, 81. p. 263-288.

Ladriere J. (1890) - « Étude stratigraphique du terrain quaternaire du Nord w. Ann. Soc. Géol. Nord. XVIII, p. 93-149.

Lautridou J.-P. (1985) - Le cycle périglaciaire pléistocène en Europe du Nord-Ouest et plus particulièrement en Normandie. Thèse de Doctorat ès Lettres, Caen, 2 vol. $908 \mathrm{p}$.

Lautridou J.-P. Sommé J. (1974) - " Les loess et les provinces climato-sédimentaires du Pléistocéne supérieur dans le NordOuest de la France x. Bull. Ass. Fr. Et. Quat., 11, p. 237-241.

Lautridou J.-P. Sommé J. Jamagne M. (1984) - « Sedimentological, mineralogical and geochimical characteristics of the ioesses of North-West France n. In - "Lithology and Stratigraphy of loess and Paleosols n, INQUA IXth Congress, Budapest. p. $121-132$

Lautrídou J.P. Sommé J. Heim J. Puisségur J - J. Rousseau D.D. (1985) - « La stratigraphie des loess et formations fluviatiles d'Achenheim (Alsace): nouvelles données bioclimatiques et corrélations avec les sèquences plèistocènes de la France du Nord-Ouest n. Bull. Ass. Fr. Et. Quat., 22, p. 125-132.

Lautridou J.-P., Monnier J.-L.. Morzadec M.-T.. Tuffreau A. (1986a) - " The Pleistocene of Northern. France $n$. Dans « Quaternary Glaciations in Northern Hemisphere 》, Quaternary Science Reviews, 5 , p. 387-393.

Lautridou J.-P., Sommé J., Heim J.. Maucorps J.. Puisségur J.-J. Rousseat D.D., Thevenin A., Van Vliet-Lanoè B. (1986b) “ Corrélation entre sédiments quaternaires continentaux et marins (littoraux et profonds) dans le domaine France septen- 
trionale-Manche n. Revue de Géologie dynamique et de Géographie physique, 27 , fasc. 2, p. 105-112

Lebret P. (1984) - La bordure nord de la province normande. évolution quaternaire, sédimentation lossique et tectonique récente. Thèse de $3^{\circ}$ cycle, Rouen, $194 \mathrm{p}$.

Léger M. (1990) - " Loess landforms o, Quaternary International, $7 / 8$, p. $53-61$.

Liu T. (ed.) (1985) - Loess and the Environment. Beijing, China Ocean Press, 251 D.

Liu T. (ed.) (1991) - Loess Environment and Global Change. Bejing. Science Press, $287 \mathrm{p}$.

Lozec V. (1964) - "Quartärmollusken der Tschechoslowakeí ». Rozpravy Ustredniho ustavu Geologikale, 31, p. 1-374.

Maher B. Thompson R. (1992) - Paleoclimatic significance of the mineral magnetic record of the Chinese Loess and Paleosols 0. Quaternary Research, 37, p. 155-170.

Mücher H.J., De Ploe J., Savat J. (1981) - « Response of loess materials to simulated translofcation by watter: micromorphologica! observations 13. Earth surface Processes and Landforms, 6. p. 331. 336.

Murton J.B., French H.M. (1992) - $\alpha$ Thaw modification of frost-fissure wedges, Richard Island, Pleistocene Mackenzie Delta, western arctic Canada 1). Jeurnal of Quaternary Science, 8. (3), p. 185 196.

Murton J.B., French H.M. (1993a) - « Thermokarst Involutions: Summer lsiand, Pleistocene Mackenzie Delta. Western Canadian Arctic 3. Permafrost and Periglacial Processes, 4, p. 217-229.

Murton J.B., French H.M. (1993b) - « Sand-wedges and permafrost history, Crumbling point, Pleistocene Mackenzie Delta, Northwest Territories, Canada 1). Proceedings of the Sixth International Conference on Permafrost, Beijing. China.

Oches E.A.. McCoy W.D. (1995) - « Aminostatigraphic evaluation of conflicting age estimates for the "Young Loess" of Hungary $\mathrm{n}$. Quaternary Research, 44, p. 160-170.

Paepe R.. Sommé J. (1970)-Les lcess et la stratigraphie du Pléistocène récent dans le Nord de la France et en Belgique, Ann. Soc. Géol. Nord, XC. p. 191-201.

Pécsi M. (1990) - $\alpha$ Loess is not just the accumulation of dust $n$. Quaternary international, 7/8, p. 1-21.

Péwé T.L. (1962) - « lce wedges in permafrost. Lower Yukon River Area, near Galena, Alaska n. Biultyn Peryglacjalny, 11, p. 65-76.

Péwé T.L. (1968) - "Loess deposits of Alaska 》. Report of the International Geological Congress 23rd. Prague, 8 p. 297-309.

Pissart A. (1987) - Géomorphologie Périglaciaire, textes et leçons de la chaire Francqui belge. Laboratoire de Géomorphologie et de Géologie du Quaternaire, Université de Liège. 135 p.

Porter S. An Z. (1995) - "Correlation between climate events in the North Atlantic and China during the Last Glaciation in. Nature, 375, p. 305-308.

Pye K. (1984) - " Loess ». Progress in Physical Geography, 8. p. 176217.

Pye K. (1987) - Aeolian dust and dust deposits. Academic press, $334 \mathrm{p}$.

Richthofen V. F. (1882) - "On the mopde of orioginof the lcess $n$. Geological magazine, 9. ser. 2, p. 293-305.

Rousseau D.D. (1987) - $\alpha$ New approach to the Pleistocene landsnails x. Loess and Periglacial phenomena, H.M. French and M. Pécsi (eds.), Budapest, Hungaricae Acaderniae Scientarum, p. 151-163.

Rousseau D.D. (1989) - u Estimation des paléotempératures hivernales en Alsace depuis 340000 ans à partir de l'analyse des associations malacologiques x. Comptes rendus de l'Académie des Sciences, 309. Sèrie II. p. 1623-1628.

Rousseau D.D. (1991) - « Climatic Transfer-function from quaternary molluscs in European Loess Deposits n. Quaternary Research. 36, p. 19-29.

Rousseau D.D., Wu N. (1997) - " A new molluscan record of the monsoon variability over the past $130000 \mathrm{yr}$ in the Luochuan loess sequence. China n. Geology, 25, p. 275-278.

Rousseau D.D., Zöller L., Valet J.-P, (1998) - u Late Pleistocene climatic variations at Achenheim. France, based on a magnetic susceptibility and TL 》. Quaternary Research, 49, p. 255-263.

Rutter N., Zongli D., Evans M.E., Yuchun W. (1990) - $\alpha$ Magnetostratigraphy of the Baofi Loess-Paleosol section in the NorthCentral China Loess Plateau n. Quaternary international, 7/8, p. $97-102$.

Smalley l.J. (1971) - " In situ theories of loess formation and the significance of the calcim carbonate content of loess x. Earth. Scien. Rev.. 7, p. 67-86.
Smalley I.J. (ed.) (1975) - Loess : lithology and genesis, Benchmark Papers in Geology 26, Dowden, Stroudsburg, Hutchinson and Ross

Sommé J. (1969) - a Stratigraphie des limons de la région du Nord de la France (Flandre-Artois) w, In : $\alpha$ La stratigraphie des lœss d'Europe 1, Suppl. Bull. Ass. Fr. Et. Quat., Congrès INQUA, Paris, p. 71-78.

Sommé J., Paepe R., Lautridou J.-P. (1980) - "Principes, méthodes et système de la stratigraphie du Quaternaire dans le Nord-Ouest de la France et la Belgigue il, Dans a Problèmes de stratigraphie quaternaire en France et dans les pays limitrophes $x_{1}$. Suppl. Bull. Ass. Fr. Et. Quat. N.S. 1. p. $148-162$.

Sornmé J., Lautridou J.-P., Heim J., et al. (1986) - " Le cycle climatique du Pléistocène supérieur dans les lœess d'Alsace à Achenheim 7. Suppl. Bull. Ass. Fr. Et. Quat.. 23, p. 97-104.

Sommé J., Munaut A.V., Etmontspohi A.F, et al. (1994) - w The Watten boring : an Early Weichselian and Holocene climatic and paleoecological record from the French North-Sea coastal plain n. Boreas, 23, p. 231-243,

Vandenberghe J., Gullentops F. (1977) - « Contribution to the stratigraphy of the Weichsel Pleniglacial in the Belgian Coversands Area n. Geol, en Mijnbouw, 56, p. 123-128.

Vandenberghe J., An Z., Nugteren G., Huayu L., Van Huissteden K. (1997), - i New absolute time scale for the Quaternary climate in the Chinese loess region by grain-size analysis $D$. Geology, 25-1, p. 35-38.

Vandenberghe J.. Pissart A. (1993) - « Permafrost changes in Europe during the last glacial y. Permafrost \& Periglacial Processes, 4, p. 121-135

Vandenberghe J. Huizer B., Mücher H. Laan W. (1998) "Short climatic oscillations in a western European leess sequence (Kesselt, Belgium) n. Journal of Quaternary Science, 13. p. $471-485$

Van Vliet-Lanoè B, (1987) - Le rôle de la glace de ségrégation dans les formations superficielles de l'Europe du Nord-Ovest. Thèse de doctorat d'État, Université Paris I. Gand, Belgique, $864 \mathrm{p}$.

Van Vliet-Lanoë B. (1990) - " Le pédocomplexe de Warneton. Oú en est-on ? Bilan paléopédologique et micromorphologique n. Quaternaire, (1), 1, p. 65-76.

Van Vliet-Lanoë B, Fagnart J.-P., Langhor R., Munaut A.V. (1992) - Importance de la succession des phases écologiques anciennes et actuelles dans la différenciation des sols lessivés de la couverture løssique d'Europe occidentale argumentation stratigraphique et archéologique v. Sciences du Sol, 30 (2). p. 75-93.

Vreeken W.J., Mücher H.J. (1981) - « (Re) deposition of loess in southern Limbourg. The Netherlands, 1-Field evidence for conditions of deposition of the lower silt loam complex n. Earth Surface Processes and Landforms, 6. p. 337-354

Wang Yong Yan W. Ten Zhi Hong T.. Le Ping Y. (1984) * Loess microtextures and the origin of loess in China 3. Lithology and Stratigraphy of loess and paleosols. M. Pésci (ed.), Geographical Research Institute, Hungarian Academy of Science, Budapest:

Washburn A. (1967) - a Instrumental observations of main wais ting in the Mister Vig district n. North East Greenland Meddel. Om Gronland, 176 (4), 303 p

Wintle A.G. (1987) - $\pi$ Thermoluminescence dating of loess $n$. Catena supplement, 9, p. 1103-1115.

Xiao J., Porter S.C.. An Z., Kumai H.. Yoshikawa S. (1995) " Grain size of quartz as an indicator of winter monsoor strenght on the Loess Plateau of Central China during the Last 130000 yr, „ Quaternary Research. 43, p. 22-29.

Zagwijn W., Paepe R. (1968) - « Die stratigraphie der weichselzeitlichen Ablagerungen der Niederlande und Belgiens y. Eiszeitalter und Gecienwart, 19, p. 129-146.

Zisheng A., Kukla G.L.. Porter C.S., Xiao J. (1991) - " Magnetic susceptibility evidence of monsoon variations on the Loess Plateau of Central China during the last 130000 years n. Quaternary Research, 36, p. 29-36.

Zöller L., Wagner G.A (1990) - « Thermoluminescence Dating of loss. Recent developments n. Quaternary international, 78, p. $119-128$.

Zöller L.. Oches E.A., Mccoy W.D. (1994) - u Towards a revised chronostratigraphy of loess in Austria with respect to key sections in the Czeck Republik and in Hungary n. Quaternary Science Reviews, 13, p. 465-472. 